\title{
Structural Dynamics Modeling of HIRENASD in Support of the Aeroelastic Prediction Workshop
}

\author{
Carol Wieseman, ${ }^{*}$ Pawel Chwalowski ${ }^{\dagger}$ and Jennifer Heeg ${ }^{\dagger}$ \\ NASA-Langley Research Center, Hampton, VA, 23681-2199 USA \\ Alexander Boucke \\ ITAM Engineering Consultants, formerly with RWTH Aachen University \\ Jack Castro \\ The Boeing Company, formerly with MSC Software Corporation
}

\begin{abstract}
An Aeroelastic Prediction Workshop (AePW) was held in April 2012 using three aeroelasticity case study wind tunnel tests for assessing the capabilities of various codes in making aeroelasticity predictions. One of these case studies was known as the HIRENASD model that was tested in the European Transonic Wind Tunnel (ETW). This paper summarizes the development of a standardized enhanced analytical HIRENASD structural model for use in the AePW effort. The modifications to the HIRENASD finite element model were validated by comparing modal frequencies, evaluating modal assurance criteria, comparing leading edge, trailing edge and twist of the wing with experiment and by performing steady and unsteady CFD analyses for one of the test conditions on the same grid, and identical processing of results.
\end{abstract}

\section{Nomenclature}

Roman Symbols

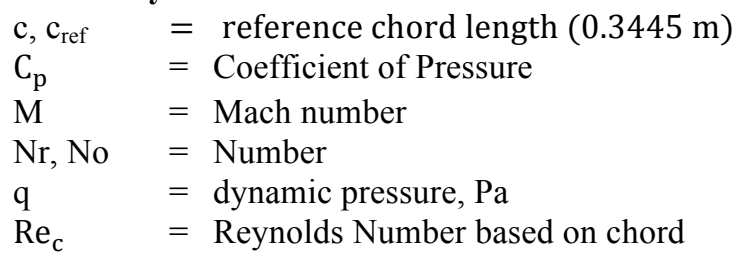

Greek Symbols

$\begin{array}{ll}\alpha & =\text { Angle of attack, degrees } \\ \omega & =\text { Frequency }- \text { radians } / \text { second } \\ \phi & =\text { Mode shape }\end{array}$

Acronyms
AePW $=$ Aeroelastic Prediction Workshop
BSCW $=$ Benchmark SuperCritical Wing
CAD $=$ Computational Aided Design
CFD $=$ Computational Fluid Dynamics
CSD $=$ Cross Spectral Density

* Senior Research Engineer, Aeroelasticity Branch, Associate Fellow, AIAA

$\dagger$ Senior Aerospace Engineer, Aeroelasticity Branch, Senior Member, AIAA

† Senior Research Engineer, Aeroelasticity Branch, Senior Member, AIAA

$\S$ Senior Scientist, ITAM Engineering Consultants, Aachen, Germany

If Structural Analysis Engineer, Boeing Commercial Airplanes

1

American Institute of Aeronautics and Astronautics 


$\begin{array}{ll}\text { DFT } & =\text { Discrete Fourier transform } \\ \text { DLR } & =\text { German Aerospace Centre } \\ \text { ETW } & =\text { European Transonic Wind Tunnel } \\ \text { FEM } & =\text { Finite Element Model } \\ \text { FRF } & =\text { Frequency Response Function } \\ \text { HEX } & =\text { Hexahedral } \\ \text { HIRENASD } & =\text { HIgh REynolds Number AeroStructural Dynamics } \\ \text { IGES } & =\text { Initial Graphics Exchange Specification } \\ \text { LE } & =\text { Leading Edge } \\ \text { MAC } & =\text { Modal Assurance Criteria } \\ \text { OML } & =\text { Outer Mold Line } \\ \text { PSD } & =\text { Power Spectral Density } \\ \text { RSW } & =\text { Rectangular Supercritical Wing } \\ \text { SPC } & =\text { Single Point Constraints } \\ \text { TE } & =\text { Trailing Edge } \\ \text { TET } & =\text { Tetrahedral }\end{array}$

\section{Introduction}

$\mathrm{T}$ HIS paper reports on a critical element required for the support of the Aeroelastic Prediction Workshop (AePW) which was held prior to the 2012 AIAA SDM conference in Hawaii.

The Aeroelastic Prediction Workshop was patterned after two very successful workshop series conducted over the past decade: the Drag Prediction Workshops ${ }^{1}$ and the High Lift Prediction Workshop ${ }^{2}$. The AePW brings together an international slate of participants to analyze a carefully selected set of unsteady aerodynamic and aeroelastic problems for which experimental validation data are available. The intent of the workshop was to investigate the ability of current computational aeroelastic tools to predict nonlinear aeroelastic phenomena, particularly those arising from the formation of shock waves and separated flow.

The AePW Organizing Committee chose three configurations for evaluation. Two configurations were rigid supercritical wings (RSW and BSCW) that had been tested in the NASA Langley Transonic Dynamics Tunnel. A summary of these configurations and results from the workshop are published in references 3-6. The third test case selected for this initial workshop was the HIgh REynolds Number Aero-Structural Dynamics (HIRENASD). ${ }^{7,8,9,10,11}$ The HIRENASD model was chosen as an initial coupled aeroelastic analysis configuration. The wing has a high degree of structural stiffness and broad spacing of the structural modes, which produces weak aeroelastic coupling and makes it a good entry-level basis of evaluation.

The first AePW workshop consisted of 59 registered attendees. Seventeen analysis teams from 10 nations provided a total of 26 analysis datasets for the three test cases consisting of 6 RSW datasets, 6 BSCW datasets, and 14 HIRENASD datasets. The workshop website contains all the presentations from the workshop ${ }^{12}$.

The HIRENASD wind-tunnel model and the loads balance were designed to be very stiff with well-separated structural modes. The first two wing-bending modes have frequencies of approximately 27 and $79 \mathrm{~Hz}$; the first wing torsion mode has a frequency of approximately $265 \mathrm{~Hz}$ experimentally. Two types of tests were conducted: angleof-attack polars and forced oscillations. The angle-of-attack polar data were obtained by varying the angle of attack at an angular sweep rate of $0.2 \mathrm{deg} / \mathrm{sec}$ while holding all other operational parameters constant.

The forced oscillation data were obtained by differential forcing of 4 piezoelectric stack exciters located at the root, at a specified frequency. The forced oscillation data to be used for the first AePW workshop were obtained by exciting near the wing's second bending modal frequency. The test cases chosen for the AePW were static cases at $\mathrm{M}=0.8, \mathrm{Re}_{\mathrm{c}}=7$ million, $\alpha=1.5 \mathrm{deg}$ and $\mathrm{M}=0.8, \mathrm{Re}_{\mathrm{c}}=23$ million, $\alpha=-1.34 \mathrm{deg}$ and forced motion of the second bending mode. An additional subsonic case at $\mathrm{M}=0.7, \mathrm{Re}_{\mathrm{c}}=7$ million $\alpha=1.5 \mathrm{deg}$ was also added; both steady aeroelastic and forced at the second bending mode frequency. The second bending mode frequency obtained experimentally wind-off was approximately $78-80 \mathrm{~Hz}$ but for the wing-only finite element models originally provided by RWTH Aachen University ${ }^{13}$, the frequency was approximately $86 \mathrm{~Hz}$. The second bending mode frequency did not vary much during wind-on testing thus indicating minimal aeroelastic coupling.

The computed modal frequencies have been published in the literature for beam and other models of the HIRENASD with some level of inconsistencies and differences. ${ }^{10,11}$ It was therefore decided to include more detail in the finite element model (FEM) to improve the correlation with experiment by making appropriate structural changes to the original solid element model to correspond better with the actual structure. 
This paper documents the changes to the HIRENASD FEM that were made during the development of a standardized FEM to be used to support the AePW, validation of the FEM development and Computational Fluid Dynamics (CFD) results using modes from the original wing-only FEM and the final modified model.

\section{Wind-Tunnel Model}

The HIRENASD is a sophisticated, semi span wind-tunnel model tested at the European Transonic Wind Tunnel (ETW) with forced oscillations of structural modes using a piezoelectric actuation system. A photo of the HIRENASD wind-tunnel model is shown in Figure 1. The wind-tunnel model is mounted vertically from the ceiling as shown. The total wind-tunnel assembly includes the wing, the wing clamping system and a wind-tunnel loads balance, exciter system and model cart.

Figure 2 shows a schematic of the mounting system, the balance and the excitation system. Forced oscillations of the model were obtained by exciting four piezoelectric stacks located near the wing root. ${ }^{11}$ The coordinate system for the parts shown is $Z$ in the direction of gravity, $X$ in the direction into the flow and $\mathrm{Y}$ in the positive lift direction.

The wing was instrumented with Stereo Pattern Tracking (SPT) for making deflection measurements. The SPT targets are also shown in Figure 1. The wind-tunnel model was instrumented with seven rows of upper and lower surface pressure transducers to measure steady and unsteady pressures and accelerometers to measure dynamic motion.

A fuselage aerodynamic fairing was installed, but was mechanically de-coupled from wing and balance, thus having no relevance for the structural model.

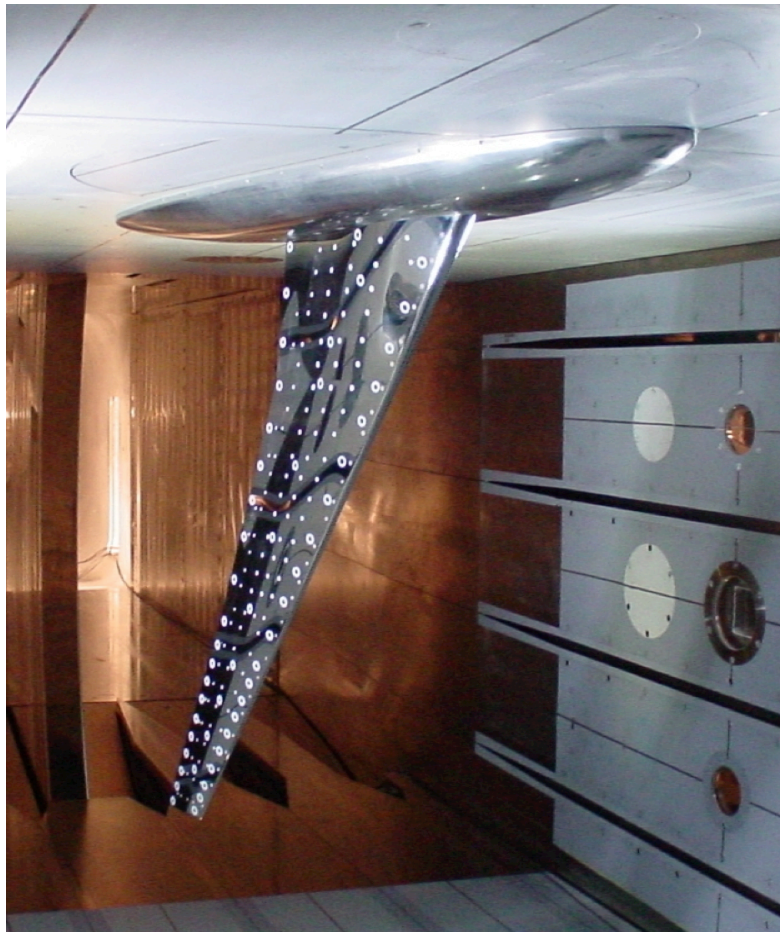

Figure 1. Photo of the HIRENASD model in the ETW.

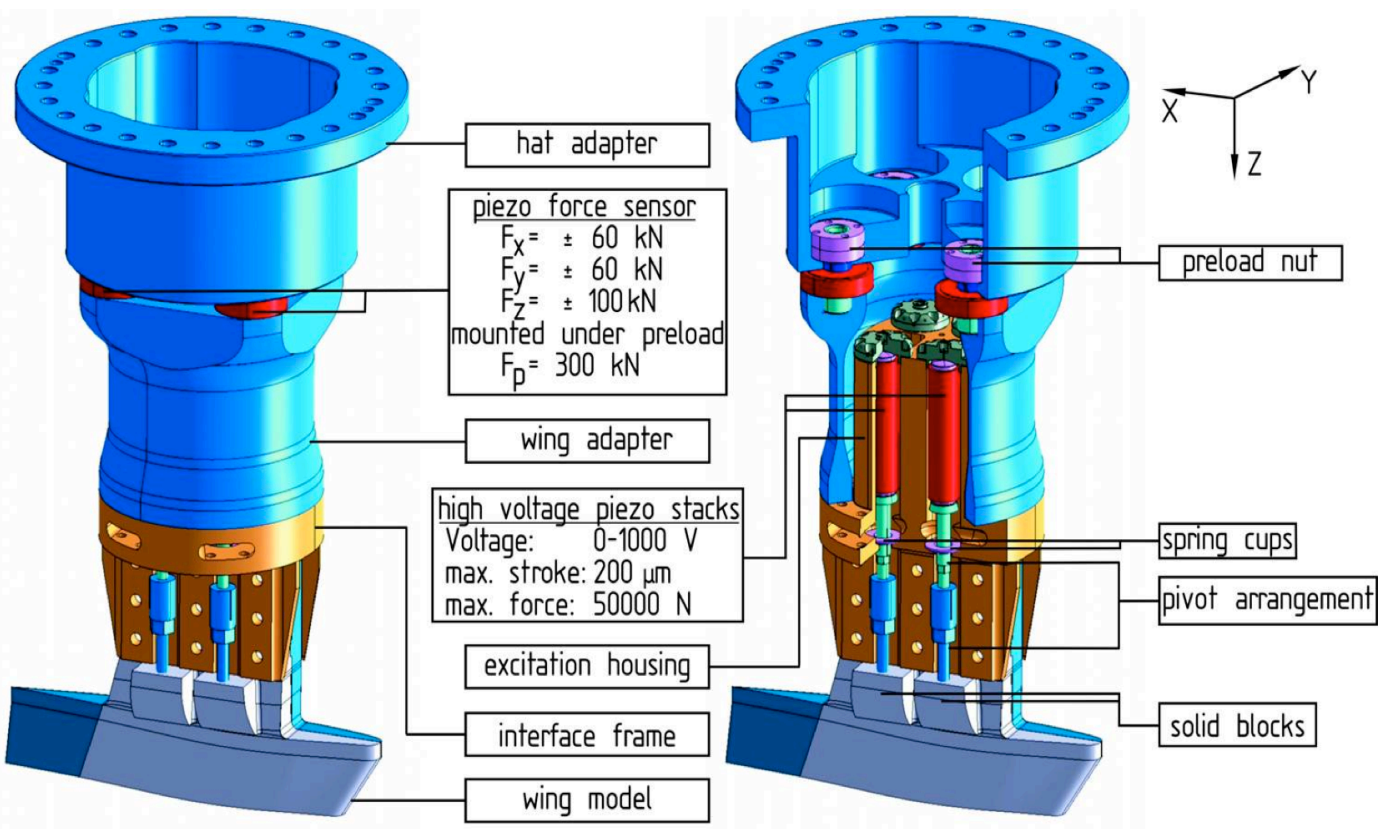

Figure 2. Schematic of mounting system, balance and excitation system. 


\section{Finite Element Model Description}

The original finite element models that were provided from RWTH Aachen University were MSC Nastran ${ }^{14}$ wing-only models shown in Figures 3 and 4 . Figure 3 shows the FEM with structured hexahedral (HEX) elements. Figure 4 shows the FEM using unstructured tetrahedral (TET) elements. The HEX wing-only model had 41,923 elements and 200,436 nodes. The TET wing-only model had 101,431 elements and 170,391 nodes.

The solid wing models that were provided had different units. The HEX model was in millimeters and the TET model was in meters. The two finite element models also had different coordinate systems. The grid locations were changed so that each model used an axis system of a right wing, $\mathrm{x}$ in the downstream flow direction, $\mathrm{y}$ out the wing span and $\mathrm{z}$ in the positive lift direction with units in meters. Figures 3 and 4 are shown in the coordinate system after the structural node locations were modified.

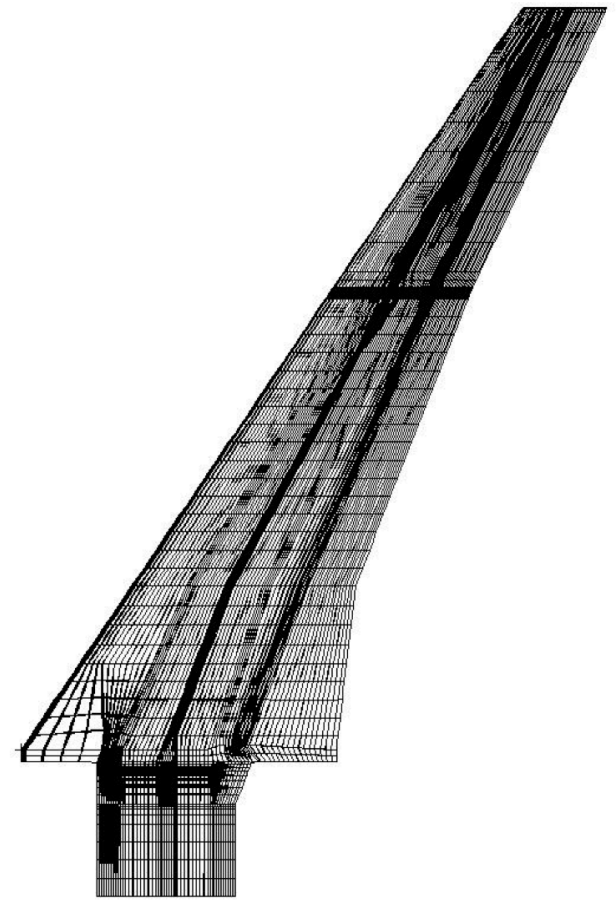

Figure 3. Wing-only FEM meshed with hexahedral solid elements.

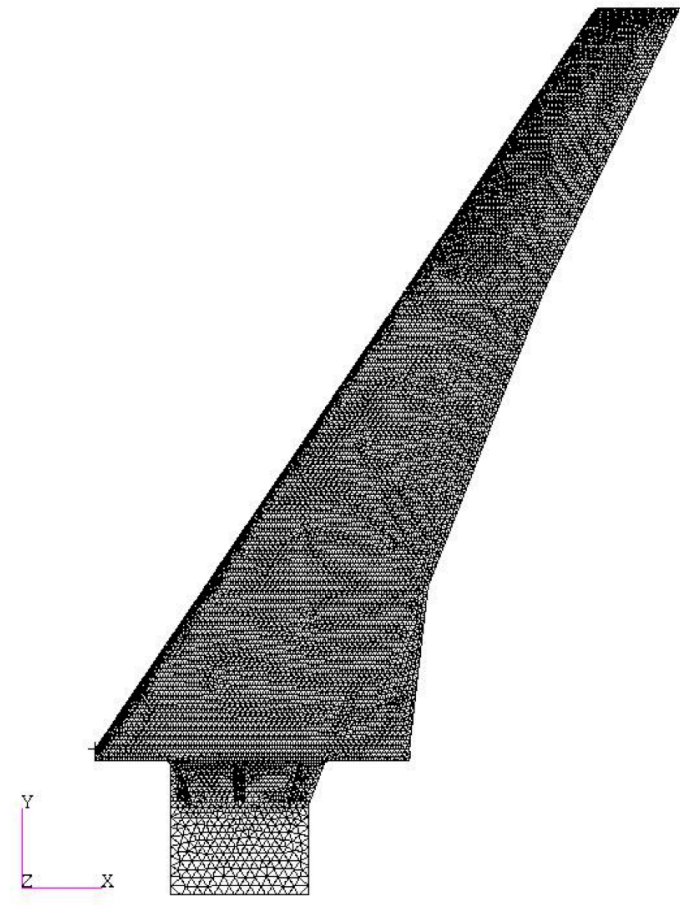

Figure 4. Wing-only FEM meshed with tetrahedral solid elements.

The second bending mode frequencies $(86 \mathrm{~Hz})$ for the wing-only models were substantially higher than the experimentally measured $78-80 \mathrm{~Hz}$ which were based on wind-tunnel test data.

This discrepancy in second bending modal frequency provided motivation for improving the FEM for use in the AePW. Questions were raised as to how these mode shapes compare and what FEM should be used for the AePW. The analysts were going to use a suite of CFD tools to analyze both static and dynamic test cases. Some were going to use fully coupled methods, i.e. structures and aerodynamics, and others just forced motion in the second bending mode. The desire to have an FEM that could be used for whatever types of tools participants chose to perform their analyses motivated the FEM improvements.

RWTH Aachen University later provided tetrahedral finite element models which included the missing parts of the mount structure, balance and the excitation elements. The first model received included just the exciter mechanism but later an FEM was received that also contained the model cart.

These models were created from CAD diagrams of each of the individual parts previously shown in Figure 2. The individual parts were linked using common grid points by equivalencing collocated nodes.

Figure 5 shows the FEM of the wing mounting system. The orientation shown is flipped top to bottom relative to Figure 2. The model cart which surrounds the assembly is not shown. The wing clamping system and exciter is shown in red. The wing root at the top of Figure 5 is black. The portion of the wing clamping structure surrounding the wing forms a "U" shape. The end of the balance closest to the wing is shown in light green, the upper section of 
the balance (in the wind-tunnel installed configuration) is shown in light blue. The piezoelectric elements in the balance are shown in black and the bolts are shown in dark blue.

Figure 6 shows the entire assembly with the wing but without the model cart. The wing is connected to the mount structure through bolts between the balance parts shown in red and the wing shown in black. Figure 7 shows the complete assembly of the wing with the balance and the model cart, from a different perspective. The part colored in cyan is the model cart. Other parts in the center of the root mounting structure are parts of the balance and exciter mechanism including the piezoelectric stacks, and bolts. The wing is shown in dark blue.

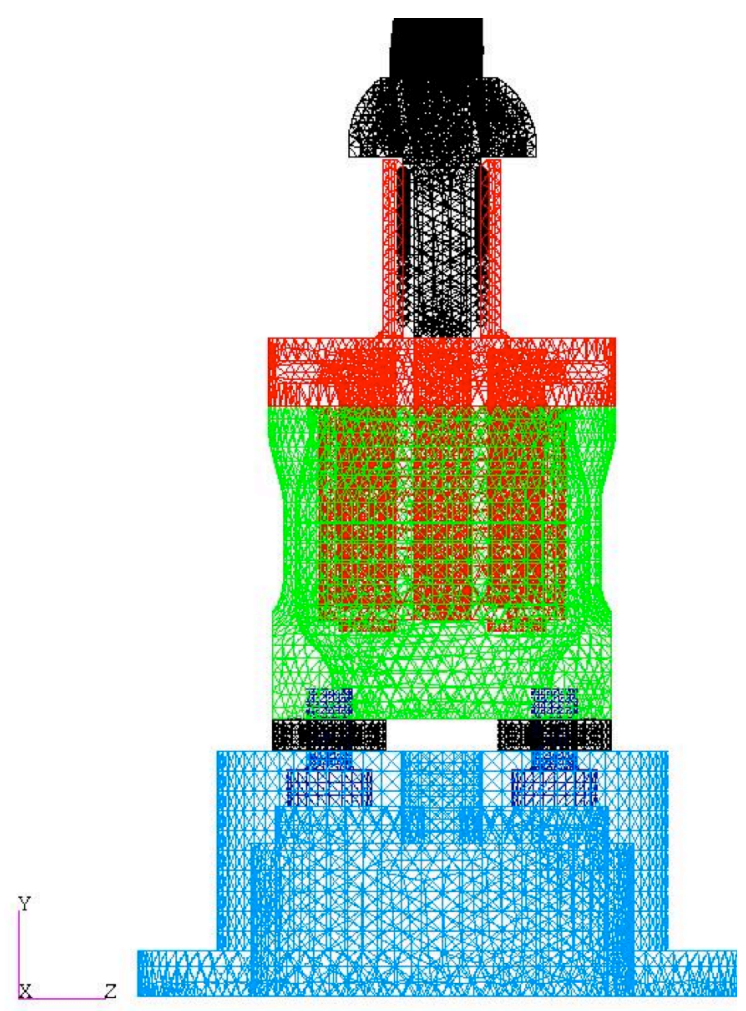

Figure 5. Wind-tunnel mount system, balance and exciter.

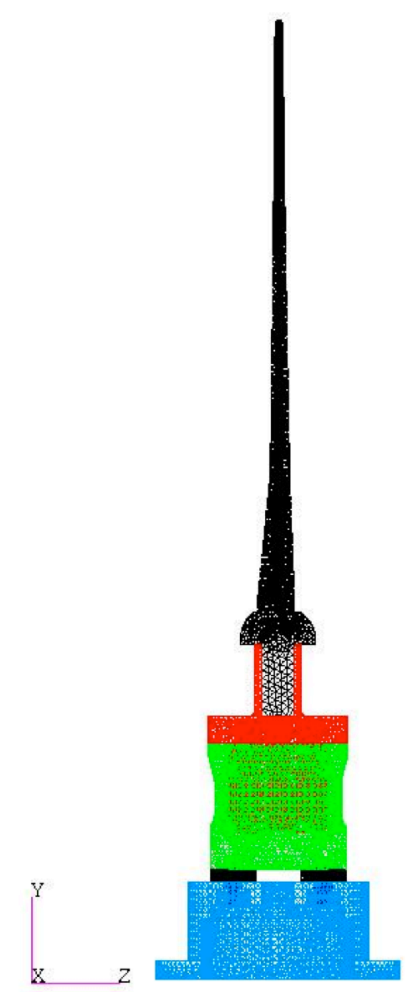

Figure 6. FEM of entire assembly without model cart. 


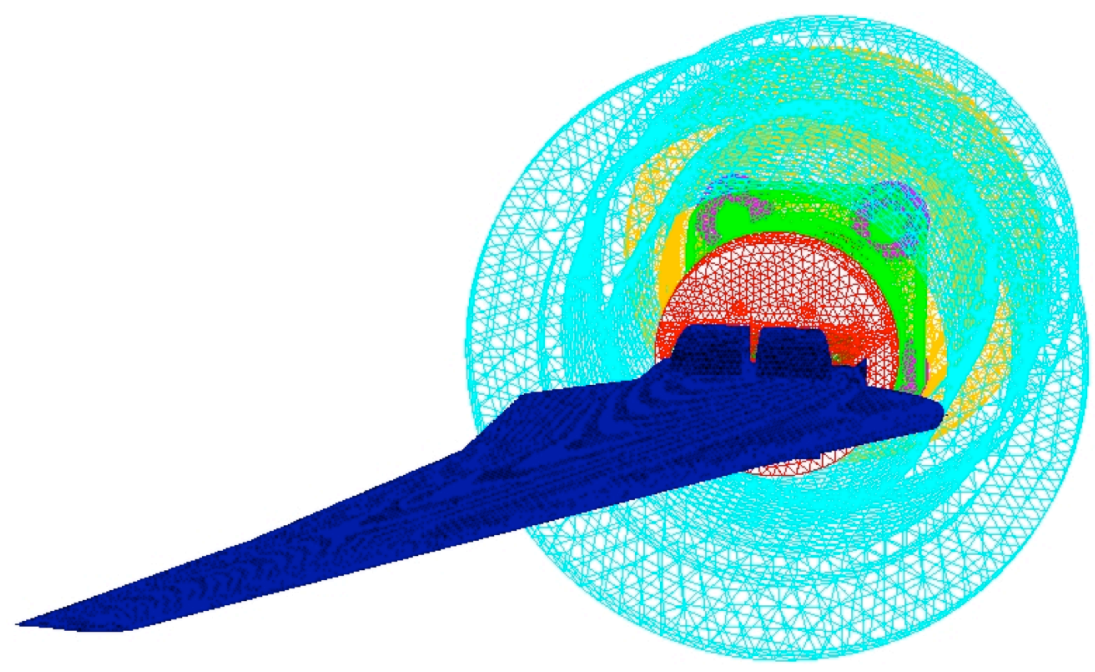

Figure 7. Complete FEM of HIRENASD including mounting structure and excitation system.

The models that were provided were modified in several steps. The first step was a modification of the wing to include instrumentation weights and associated cabling. Modifications were made to be able to output displacements at accelerometer locations. The connection of the wing to the balance assembly was then modified. The final modification was the modification of the structural Outer Mold Line to correspond to the OML of the Initial Graphics Exchange Specification (IGES) wing used for the CFD analyses. The details of these modifications are described next.

\section{A. Modeling of concentrated masses for wiring and instrumentation}

Enhancements of the FEM included adding concentrated masses for wiring and instrumentation. Figure 8 shows a photo with the location of the instrumentation. Instrumentation weights and their location were provided by RWTH Aachen University and were applied as concentrated masses and then linked to grid points in the FEM as shown in Figure 9.
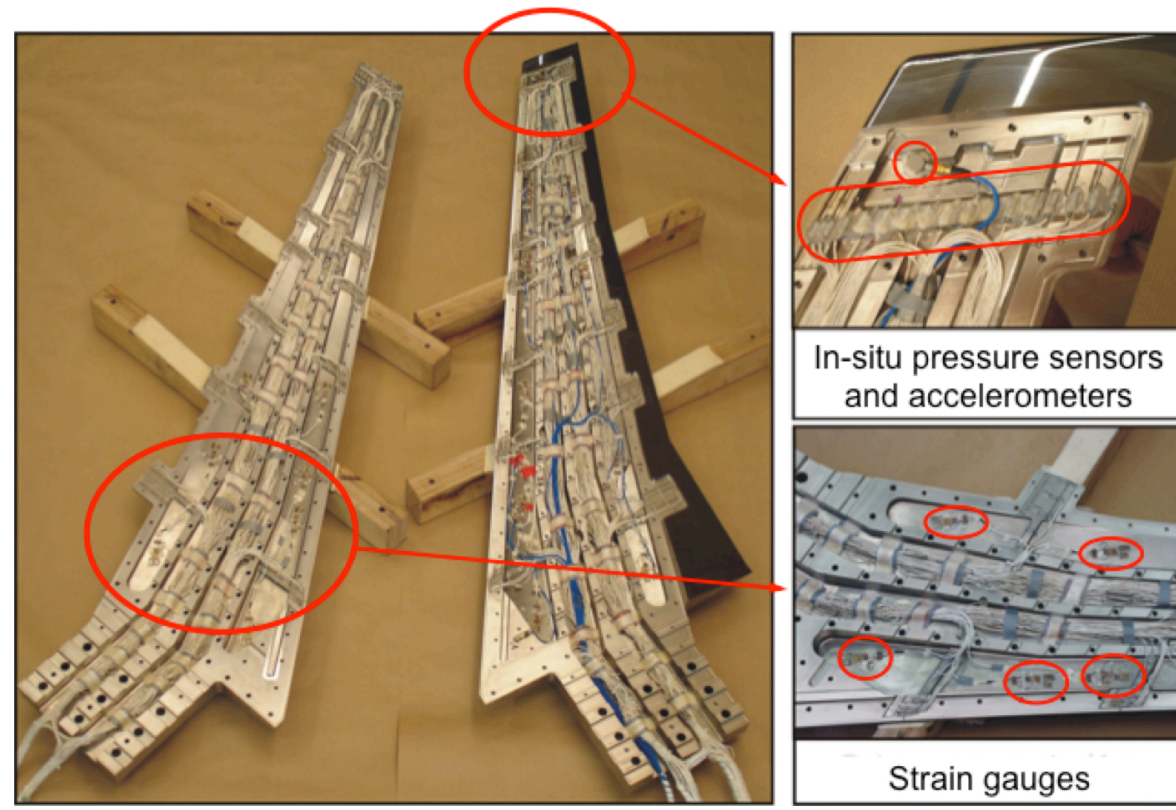
and accelerometers

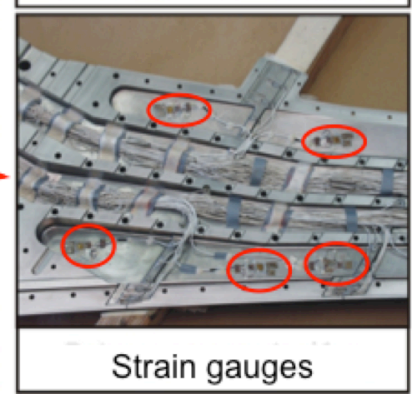

Figure 8. Wind-tunnel model showing strain gauges, internal wiring and pressure transducers.

American Institute of Aeronautics and Astronautics 


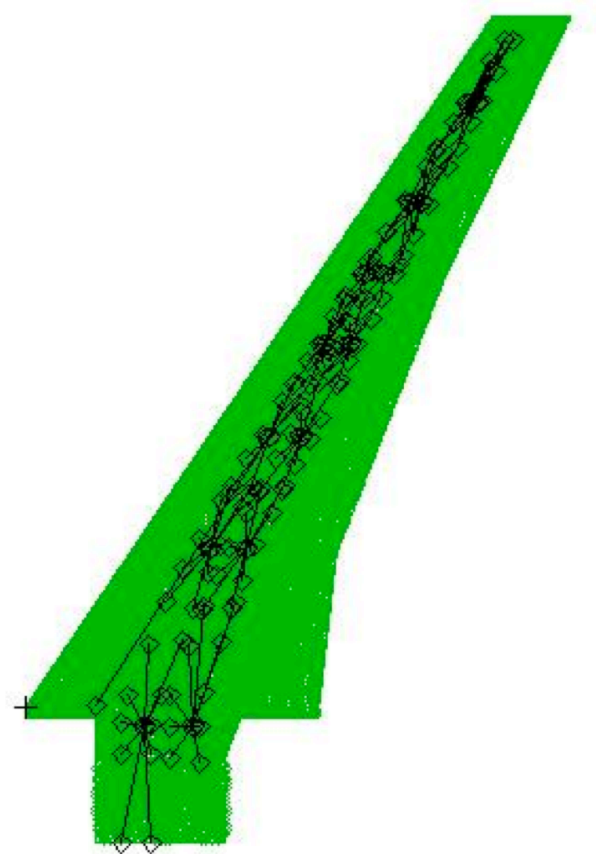

Figure 9. FEM Model with instrumentation wiring included.

\section{B. Measurements at Accelerometer Locations}

Measurements at the accelerometer locations in the FEM were required in order to compare the mode shapes of the FEMs with experimental data. There were 11 wing accelerometers, however, only 10 measured motion in the out-of-plane direction. These are shown in the schematic of Figure 10. Accelerometer Nr 12/3 measured the acceleration in an in-plane direction. Accelerometer $\mathrm{Nr} 11 / 2$ was bad during experimental testing so only nine accelerometers were available to resolve experimental mode shapes.

Figure 11 shows the FEM of the wing with nodes located at the accelerometer locations. All ten locations are shown including the one that was bad. The first attempt to obtain the analytical measurements at each accelerometer location was to use the closest grid in the FEM. It was more accurate to add grids at the precise accelerometer locations and then use Rigid Body Elements to average the displacements from multiple grid locations close to each accelerometer location to get the actual displacement at the accelerometer location. Therefore, the grid numbers for the accelerometer locations were independent of the structural model and the locations were more accurate. The lines connecting the accelerometers are non-structural elements so that the mode shape can be viewed in MSC Patran ${ }^{14}$ using just the displacements at the accelerometer locations. 


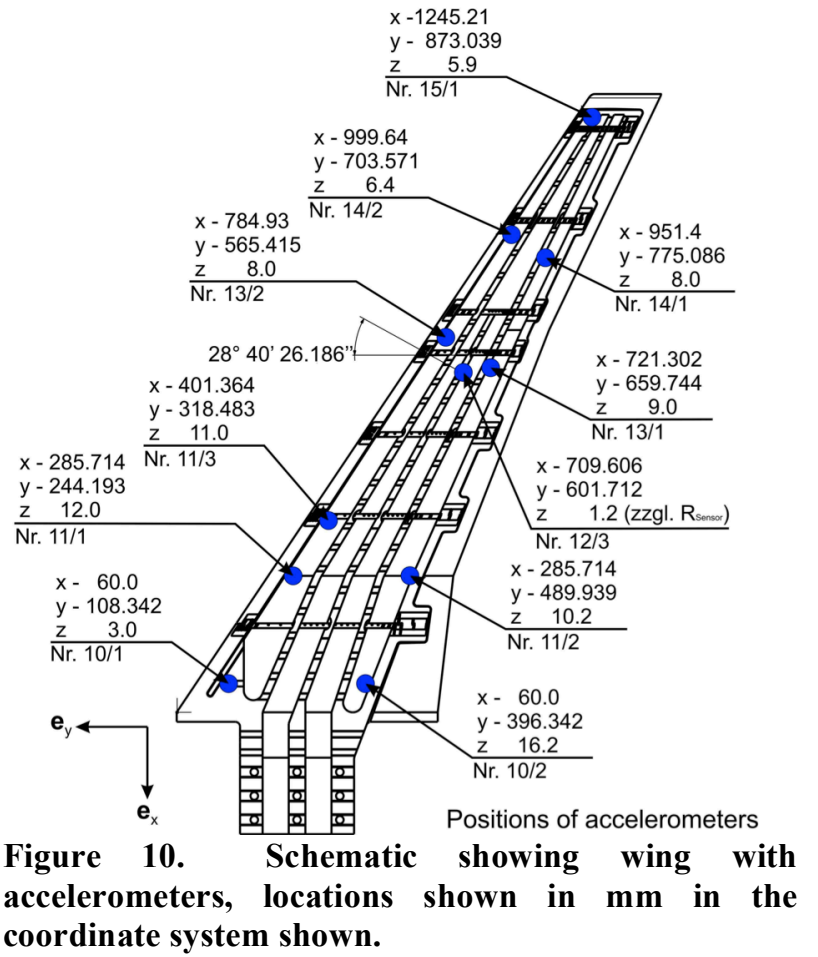

Figure 10. Schematic showing wing with coordinate system shown.

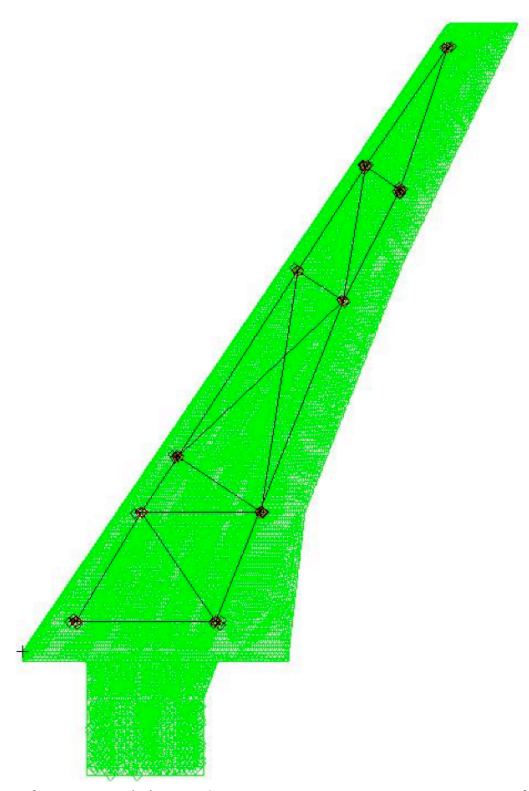

Figure 11. Accelerometer modeling in FEM.

\section{Modifications of Connection of Wing Root to Exciter}

The wing-only FEMs had cantilevered boundary conditions at the wing root corresponding to the bottom of the "U" shown in Figure 5. However, the physical connection of the wing to the balance structure is through bolts under plates. The bottom of the "U" was not connected between the wing and the additional structure except through friction. The original FEM including the mount structure used equivalencing of collocated nodes to connect the two parts. To improve the modeling of this connection in the FEM, the connection of the wing to the wing clamping structure was modified.

Figure 5 shows the wing mounting assembly and part of the wing. The connection of the wing root to the balance assembly was originally done using equivalencing of nodes in the entire U-shaped section that is the intersection between the wing in black and the exciter system in red. The wing was disconnected from the balance by creating duplicate grid points at the same locations as those that had been equivalenced and recreating the solid element cards using these grid points. This corresponds to the U-shaped section in the more complex FEMs.

Single point constraints (SPC) were used to connect the wing and the balance at all the locations that had been equivalenced to match the original model. This was done for verification purposes. MATLAB ${ }^{15}$ based tools were developed to facilitate the FEM modification and rewrite the Nastran input deck.

To better capture the wing root boundary conditions, the base of the wing was disconnected entirely at the wing root attachment point or identified at the base of the U shown in previously shown in Figure 5. The SPC's associated with the bottom of the "U" were removed and the only SPC's which were kept were those located at the bolt locations shown in Figure 12 as shown in dark blue. These are also seen in the isometric view in Figure 13 connecting the clamping structure/exciter (red) and the wing (black). 


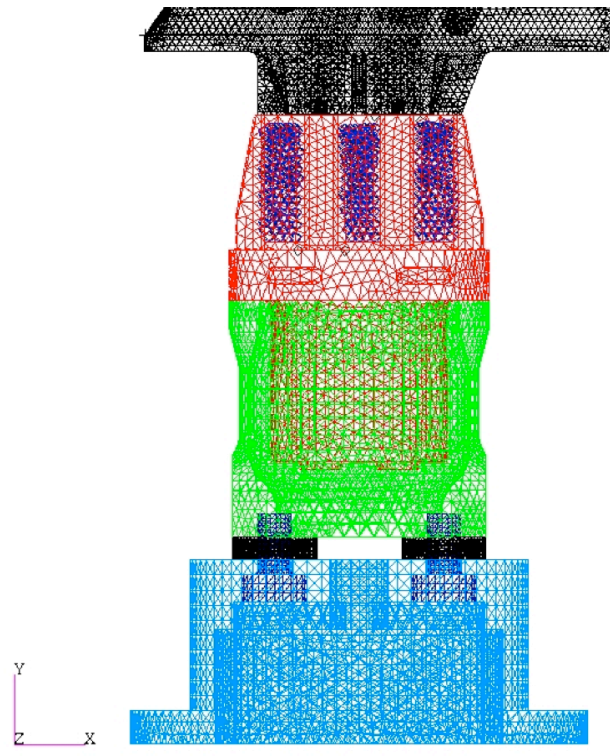

Figure 12 FEM of clamping mechanism, exciter and balance assembly.

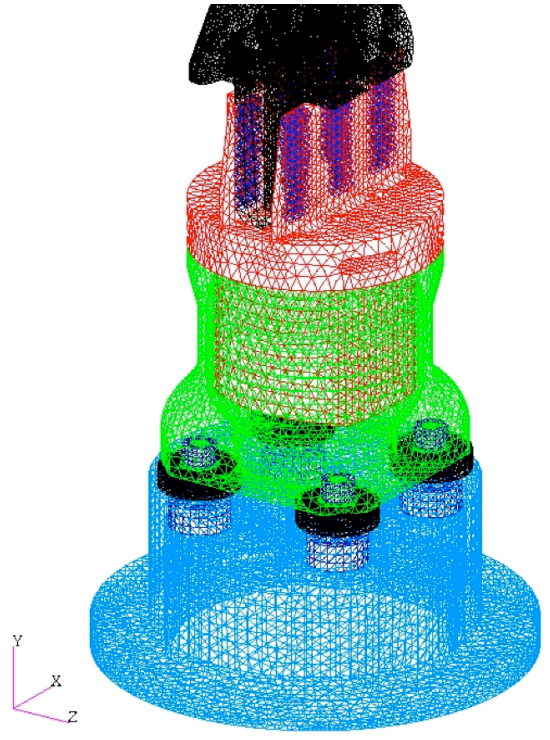

Figure 13. Isometric view of clamping mechanism, exciter and balance assembly.

\section{Modification to Structural OML}

Several of the analysts participating in the AePW used fully coupled structural and aeroelastic solutions, requiring the structural and the aerodynamic models to have identical outer mold line (OML) geometry. The presentations of all the analysts that include the details of how they applied the FEM are available on the AePW Website. $^{12}$

The wing FEM OML did not match the IGES OML used for gridding for the aerodynamics. The solution was to project the wetted surface TET nodes to the IGES surfaces using a perpendicular projection to the closest surface (Patran: Modify/Node/Project function). ${ }^{14}$ There were some difficulties in that a few interior nodes were also included and projected. Figure 14 shows the TET wing model and a few of the elements that include these interior nodes that were projected to the IGES OML. Most of these elements were near the wing tip. A couple of examples are shown in the figure. The projection of these interior nodes resulted in elements which were no longer tetrahedrals but were planar. The solution was to identify the interior nodes which were projected and move them back to their original locations.

There was an inconsistent definition of the "trailing edge" in the FEM model as compared with the IGES geometry which was not resolved. A comparison of the trailing edge of the FEM and the IGES model are shown in Figure 15. The FEM trailing edge shown in red has no thickness, whereas the IGES model in green has finite thickness. 


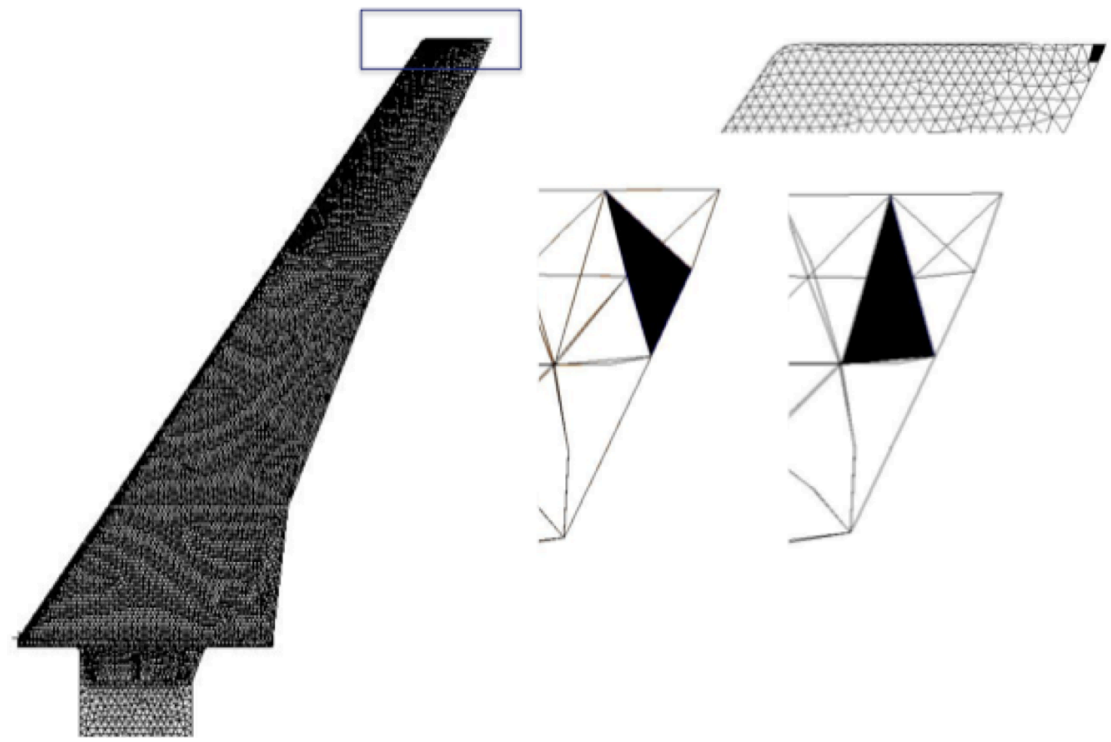

Figure 14. Issues with projection of the grids to the IGES OML.

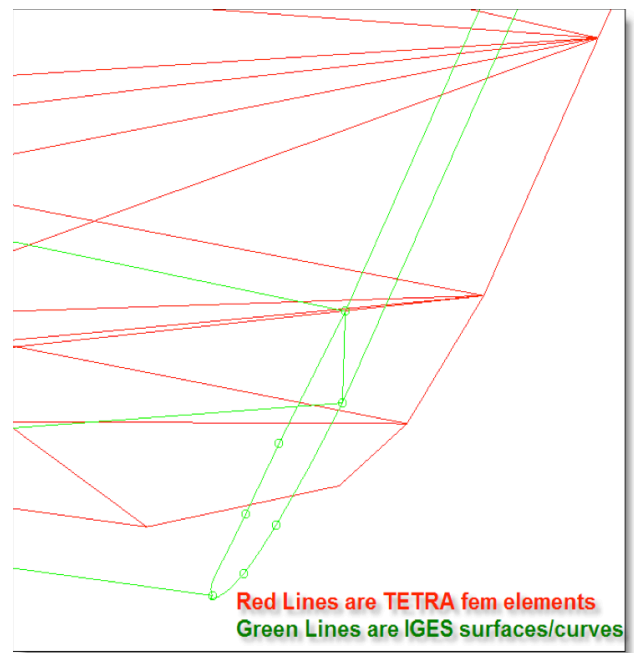

Figure 15 Comparison of trailing edge of FEM model and the IGES surfaces and curves.

\section{Validation of the Finite Element Model Changes.}

The finite element model changes were validated by comparing modal frequencies, modal assurance criteria, leading edge and trailing edge deflections, twist distribution, node lines and displacements at the accelerometer locations from FEM analyses and experimental results.

\section{A. Modal Frequencies}

A comparison of the modal frequencies is shown in Table 1. The first column is an identification of the predominant shape of the mode. "B" signifies out-of-plane bending mode. "T" signifies torsion mode. "FA" is a inplane fore-and-aft mode that can't be resolved by the experiment because the accelerometers measure out-of-plane motion. 
Table 1. Modal frequencies for different FEM's compared with experiment.

\begin{tabular}{|c|c|c|c|c|c|c|c|c|c|c|}
\hline & Case 1 & Case 2 & Case 3 & Case 4 & \multirow{3}{*}{$\begin{array}{l}\text { Case } 5 \\
\text { Add exciter } \\
\text { but no } \\
\text { modelcart }\end{array}$} & \multirow{3}{*}{\begin{tabular}{|c|} 
Case 6 \\
Add \\
modelcart
\end{tabular}} & \multirow{3}{*}{\begin{tabular}{|l|} 
Case 7 \\
\\
Add instr. \\
\end{tabular}} & \multirow{3}{*}{\begin{tabular}{|c|} 
Case 8 \\
$\begin{array}{c}\text { Disconnect } \\
\text { bottom of } \\
\text { "U" }\end{array}$ \\
\end{tabular}} & \multirow{3}{*}{\begin{tabular}{|l|} 
Case 9 \\
$\begin{array}{l}\text { Mod at bolt } \\
\text { connection }\end{array}$ \\
\end{tabular}} & \multirow{3}{*}{\begin{tabular}{|c} 
Case 10 \\
\\
Projected \\
OML
\end{tabular}} \\
\hline & \multicolumn{2}{|c|}{$\begin{array}{c}\text { Experimental } \\
\text { Frequencies }\end{array}$} & \multicolumn{2}{|c|}{ Wing only models } & & & & & & \\
\hline & ARTeMIS & Analyzer & HEX 20 & HEX 8 & & & & & & \\
\hline $1 \mathrm{~B}$ & 26.015 & 26.250 & 26.541 & 26.534 & 26.249 & 26.217 & 25.618 & 25.604 & 25.542 & 25.550 \\
\hline $2 \mathrm{~B}$ & 78.635 & 78.203 & 86.019 & 85.932 & 82.881 & 82.257 & 80.812 & 80.688 & 80.199 & 80.245 \\
\hline $1 \mathrm{FA}$ & & & 156.938 & 157.237 & 117.465 & 110.904 & 108.626 & 106.998 & 106.242 & 106.193 \\
\hline $3 B$ & 166.250 & 166.250 & 189.311 & 189.434 & 170.083 & 163.745 & 161.770 & 161.441 & 160.381 & 160.349 \\
\hline $4 \mathrm{~B}$ & 245.002 & 245.000 & 321.774 & 321.985 & 259.317 & 244.899 & 242.520 & 242.364 & 241.942 & 241.995 \\
\hline $1 \mathrm{~T}$ & 265.855 & 265.781 & 272.859 & 273.443 & 275.120 & 273.055 & 272.295 & 272.182 & 271.718 & 271.844 \\
\hline $2 T$ & & & 450.506 & 451.811 & 448.517 & 443.496 & 442.291 & 441.178 & 437.122 & 437.830 \\
\hline $5 B$ & & & 496.680 & 497.795 & 380.224 & 360.338 & 356.863 & 356.332 & 354.341 & 354.155 \\
\hline $2 \mathrm{FA}$ & & & 422.976 & 423.259 & 280.260 & 256.158 & 252.790 & 252.651 & 252.361 & 252.225 \\
\hline $3 F A$ & & & & & 499.387 & 454.750 & 450.171 & 446.396 & 444.318 & 443.805 \\
\hline $3 T$ & & & 622.407 & 625.227 & & & & & & 569.737 \\
\hline $6 \mathrm{~B}$ & & & & & & & & & & 497.802 \\
\hline $3 T$ & & & & & & & & & & 569.737 \\
\hline 7TB & & & & & & & & & & 643.234 \\
\hline
\end{tabular}

Cases 1 and 2 are the experimental modal frequencies calculated using two different methods. Wind-off modal survey data was acquired for the HIRENASD by hammer impact test and by frequency sweeps of the piezoelectric stack actuators. ${ }^{11}$ However, the modeshapes and the data acquired during those tests were not available. The only dataset available for extracting mode shapes was acquired by exciting the HIRENASD wind-tunnel model with a 35 $\mathrm{Hz}$ excitation of the piezoelectric stack actuators. This frequency was chosen because it is not near a modal frequency or a harmonic or subharmonic of a modal frequency. Data sets obtained using excitation near a presumed modal frequency would tend to force the system to respond at the excitation frequency rather than the system natural frequency. RWTH Aachen University provided frequencies and the five experimental modeshapes extracted using ARTeMIS, Ambient Response Testing and Modal Identification Software. ${ }^{16}$ The time histories of the nine accelerometers were also available.

Since thorough understanding was desired as to how these modal frequencies and mode shapes were determined, independent calculations were done using a NASA MATLAB based package named TDT_analyzer. ${ }^{17}$

The mode shapes for the TDT_analyzer modes were determined by calculating the frequency responses of all the accelerometers with reference to the outboard wing tip accelerometer identified as $\mathrm{Nr} 15 / 1$. This was the accelerometer that would later be used by the CFD analysts to calculate the frequency responses of the pressure distributions and is shown in Figure 10. The FRFs were extracted at the modal frequencies as determined from the Power Spectral Density (PSD's) of the excitation signal. A third method was also investigated using the Complex Mode Indicator Function ${ }^{18}$ (CMIF) which relies on Singular Value Decomposition. No details or results are presented for this method in this paper.

Case 3 shows the original wing-only modal frequencies of the first 10 flexible modes of the wing-only solid element model identified as HEX20. Due to the computational time the FEM was modified to use HEX8 elements which did not include the intermediate nodes on the edges that are defined in HEX20 elements. This reduced the size of the finite element model and decreased the time required to calculate the mode shapes. The modal frequencies corresponding to the HEX8 model are shown as case 4. This is the model that will be compared with the final modified model later in the paper and will be identified in the rest of the paper as the "Original Model".

The AePW team was also provided with the solid element wing-only model that used tetrahedral meshing which yielded frequencies virtually identical to the HEX20 model and therefore are not shown in the table.

Case 5 shows the modal frequencies for the tetrahedral solid element model of the wing with the addition of the tetrahedral models of the balance and exciter system. There was a substantial reduction in the second bending mode from about $86 \mathrm{~Hz}$ to approximately $83 \mathrm{~Hz}$ by adding the balance.

The model-cart was then added (Case 6) resulting in a further reduction of the second bending mode frequency. Case 7 included the addition of the masses for instrumentation and wiring.

When the FEM was constructed, each structural section was meshed and collocated nodes were equivalenced. The junction between the exciter and the model cart did not accurately represent the physical connection so the boundary condition was modified and the nodes disconnected between the exciter and model-cart at the base of the U-shaped section of the wing clamping and excitation system and is shown as Case 8. 
Case 9 shows the impact of disconnecting the grids between all the grids on the U-shaped section connecting the wing to the wing clamping and excitation system and then adding constraints at those locations where the bolts at are located as shown in Figure 12.

Finally because of the requirement for different analysts to have a finite element model that had a consistent outer mode line structurally and aerodynamically the structural model was projected to the OML of the aerodynamics model and these results are shown in Case 10.

\section{B. Modal Assurance Criteria}

The impact of the changes in the FEM was also investigated using modal assurance criteria (MAC) ${ }^{19}$. Modal assurance criteria is a widely used technique to estimate the degree of correlation between mode shape vectors. The MAC is often used to pair mode shapes derived from analytical models with those obtained experimentally. It is easy to apply and doesn't require any estimate of the system matrices. The MAC between a mode $j$ from model or experiment " $m$ " with mode $k$ from analytical model " $a$ " is defined as:

$$
M A C_{j k}=\frac{\left|\phi_{m j}^{T} \phi_{a k}\right|^{2}}{\left(\phi_{a k}^{T} \phi_{a k}\right)\left(\phi_{m j}^{T} \phi_{m j}\right)}
$$

The MAC varies from 0 to 1 . A value of 1 shows that the mode shapes are correlated well. If the modes are all orthogonal then the MAC is 1 for all the diagonal terms and 0 for the off diagonal terms when comparing a model to itself.

In the case of the experimental wind-tunnel test, nine working accelerometers were used to measure time histories while exciting the second modal frequency thereby obtaining the experimental mode shapes. The only data available to compare the experimental modes with the analytical modes were these nine accelerometers. A tenth accelerometer on the wind-tunnel model was bad. This bad accelerometer, $\mathrm{Nr} 11 / 2$, was located near the inboard trailing edge as shown in Figure 10.

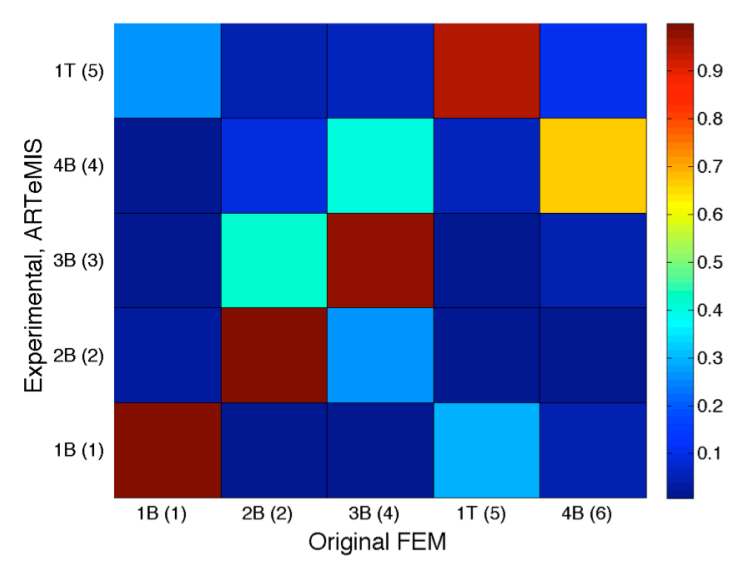

Figure 16. MAC Original model compared with experiment.

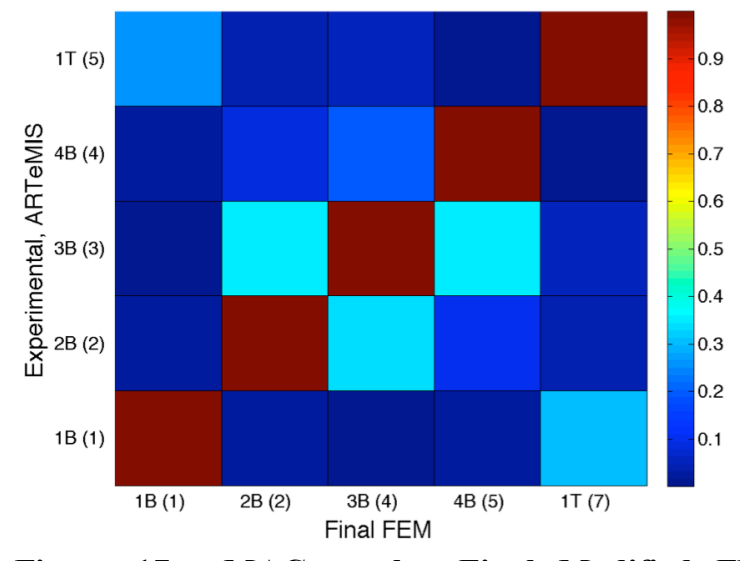

Figure 17. MAC results, Final Modified FEM compared with experiment.

MAC was used to compare the experimental results with the analytical modal results calculated by the Nastran analyses and for comparing the different FEMs to each other. Figure 16 shows the MAC results for the Original structural model compared with experimental mode shapes. The left side shows the experimental modal descriptions, the bottom shows the analytical modal descriptions and the Nastran mode number. The fore-aft modes have been removed in these comparisons. Note that the fourth bending mode found in the experiment is not well correlated with the finite element mode of the Original FEM. Note that for the final FEM, the fourth bending mode (4B) has moved from $\# 6$ to $\# 5$ and the first torsion (1T) mode has moved from $\# 5$ to $\# 7$. Figure 17 shows the MAC results for the final modified FEM compared with experiment. The result is that the 4B and 1T modes correlate better with experiment without corrupting the correlation for the 1B, $2 \mathrm{~B}$ and $3 \mathrm{~B}$ modes. Figure 18 shows the MAC 
results comparing the original model with the final modified model. This figure indicates the extent to which the mode shapes were changed during the modification process. During the modification process the MAC was evaluated between each FEM and experiment and between each successive FEM.

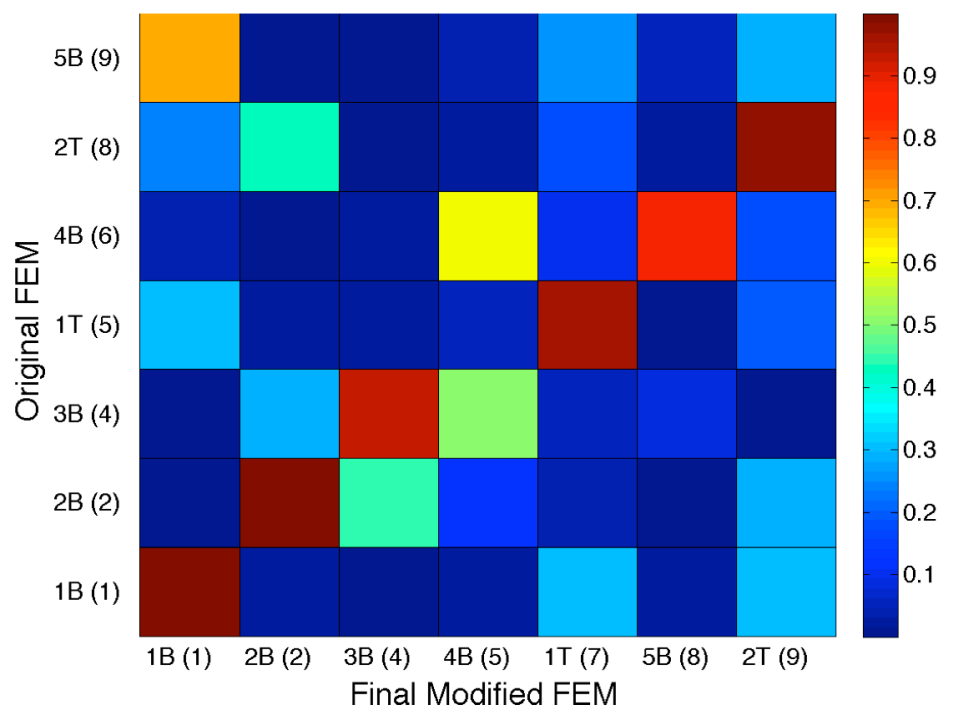

Figure 18. MAC Results, Original FEM compared with Final Modified FEM.

\section{Comparison of Second Bending Mode Shape}

Additionally the second bending mode shape was compared by comparing the vertical displacements at the wing leading and trailing edges and the wing twist. The node line was also compared.

The mode shape deflections of ARTeMIS modes were normalized such that $\phi_{n}^{T} \phi_{n}=1$ using the nine accelerometers. In order to compare the analytical mode shape with these experimental mode shape, the analytical second bending mode shape was scaled in the same manner.

Although the output of the finite element model had mode shapes all scaled to unit generalized mass, the second bending mode shape was scaled for comparison with experimental data so that $\phi_{n}^{T} \phi_{n}=1$ for that mode. This meant that the amplitude of each displacement was scaled by

$$
\text { GMfactor }_{n}=\sqrt{\frac{1}{\phi_{n}^{T} \phi_{n}}}
$$

For the Original FEM the factor for the second bending mode was 1.666 and for the final modified model the factor was 1.763 which corresponds to almost a $6 \%$ difference. This would impact comparison of the leading edge and trailing edge deflections and wing twist in comparing analytical mode shapes with each other using the nine accelerometers. The deflections of the experimental mode shapes calculated using TDT_analyzer were also modified by the same formula which corresponded to 0.711 . The calculated modeshapes using TDT_analyzer were normalized by the displacement at the outboard accelerometer ( $\mathrm{Nr} 15 / 1)$.

\section{Vertical Deflections at Leading Edge and Trailing Edge, and Wing Twist}

As stated previously, the only data available from the experiment were the displacements at the nine accelerometer locations. The second bending mode shape was interpolated and extrapolated to uniformly spaced points using the MATLAB griddata function to the entire wing area. The leading edge and trailing edge deflections and wing twist were calculated from these interpolated mode shapes and compared with analytical mode shapes interpolated to the planform in the same manner. 
The results shown in this paper compare the original FEM and the final modified FEM but each successive change in the model was also compared in the same manner with experiment and previous FEMs.

A comparison between experimental and FEM results for wing leading edge deflections is shown in Figure 19. A comparison between experimental and FEM results for vertical displacement at the trailing edge is shown in Figure 20. The corresponding twist is shown in Figure 21. The final modified FEM was closer to the experimentally measured mode shape than the original FEM was. The mode shapes are very similar, with the Final Modified FEM second bending mode shape showing an improved correlation to the experimental mode shape.

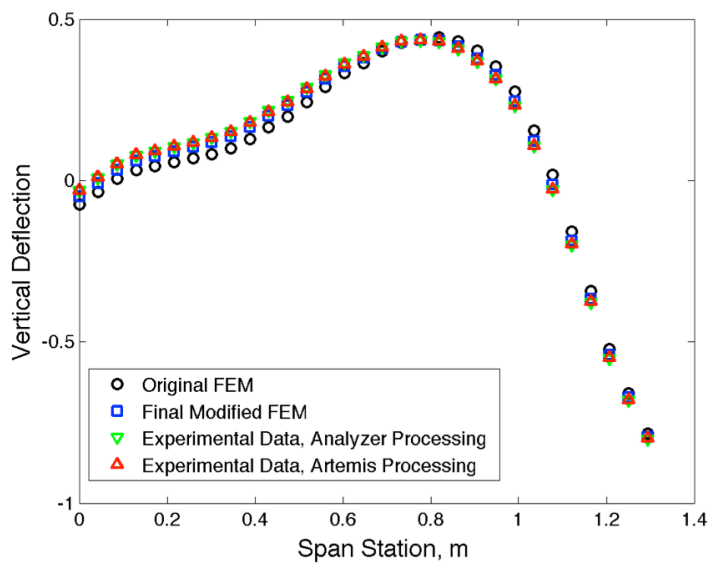

Figure 19. Comparison of vertical deflection at LE.

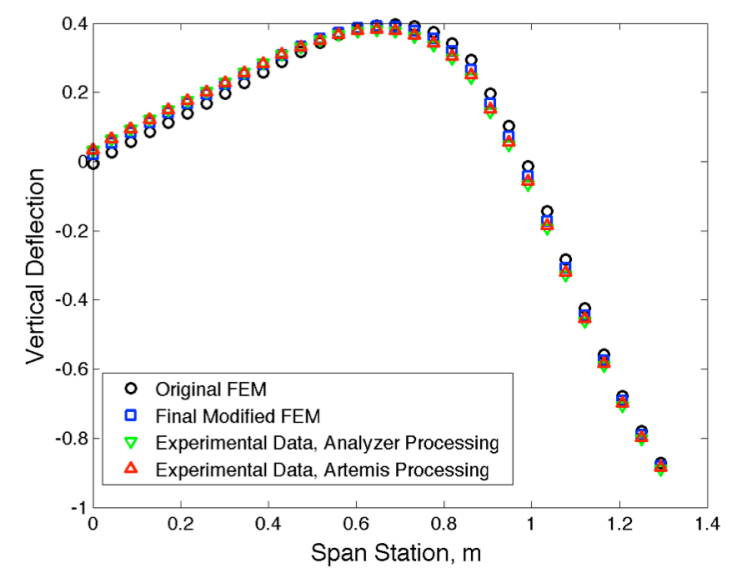

Figure 20. Comparison of vertical deflection at TE.

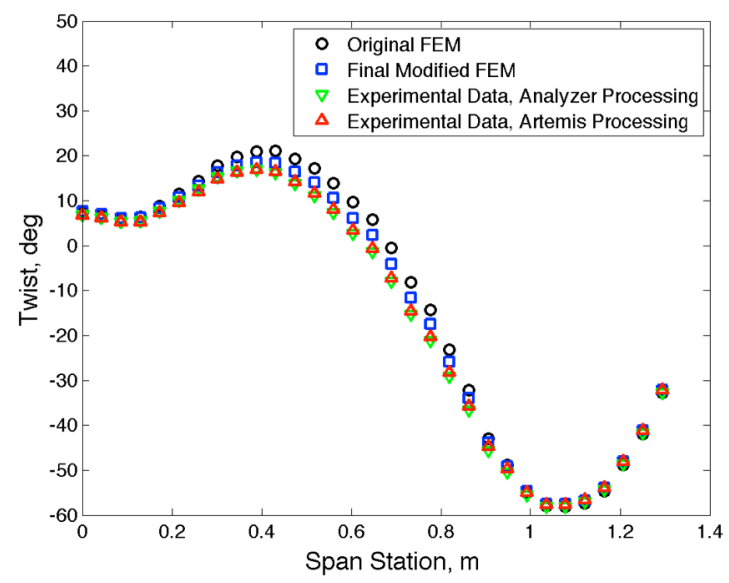

Figure 21. Comparison of Wing Twist.

\section{Node Lines}

The node lines were also compared between the experimental results and the finite element models using the same interpolated/extrapolated data. The experimental results are shown in Figure 22. The original FEM node line is shown in Figure 23 and the Final Modified FEM node line is shown in Figure 24. The node lines lie between the magenta squares and the green circles. The red circles correspond to the locations of the accelerometers used for the interpolation and extrapolation of the mode shape. There are small differences in the node line that can be discerned even with the meshgrid distribution shown. 


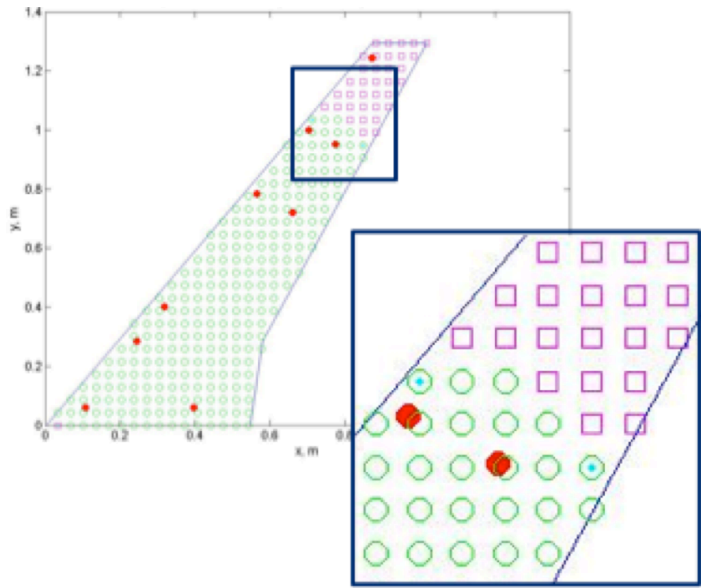

Figure 22. Node line of experimental data.

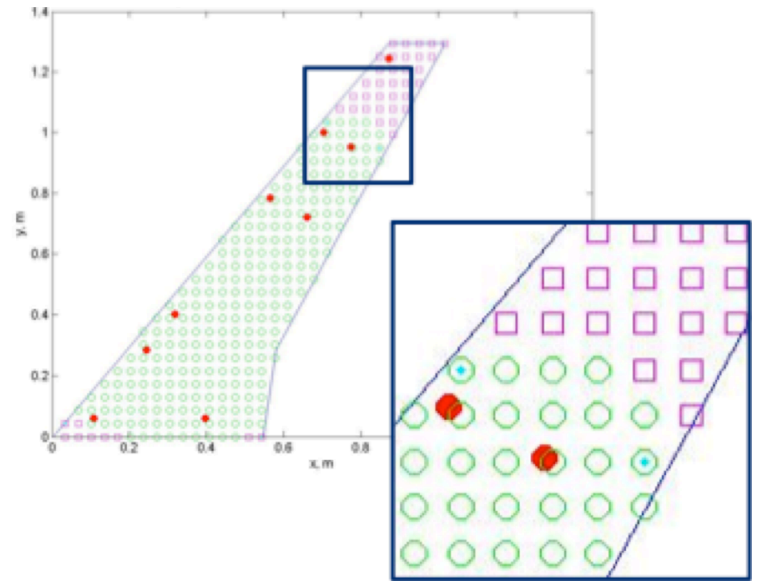

Figure 23. Node line of original FEM.

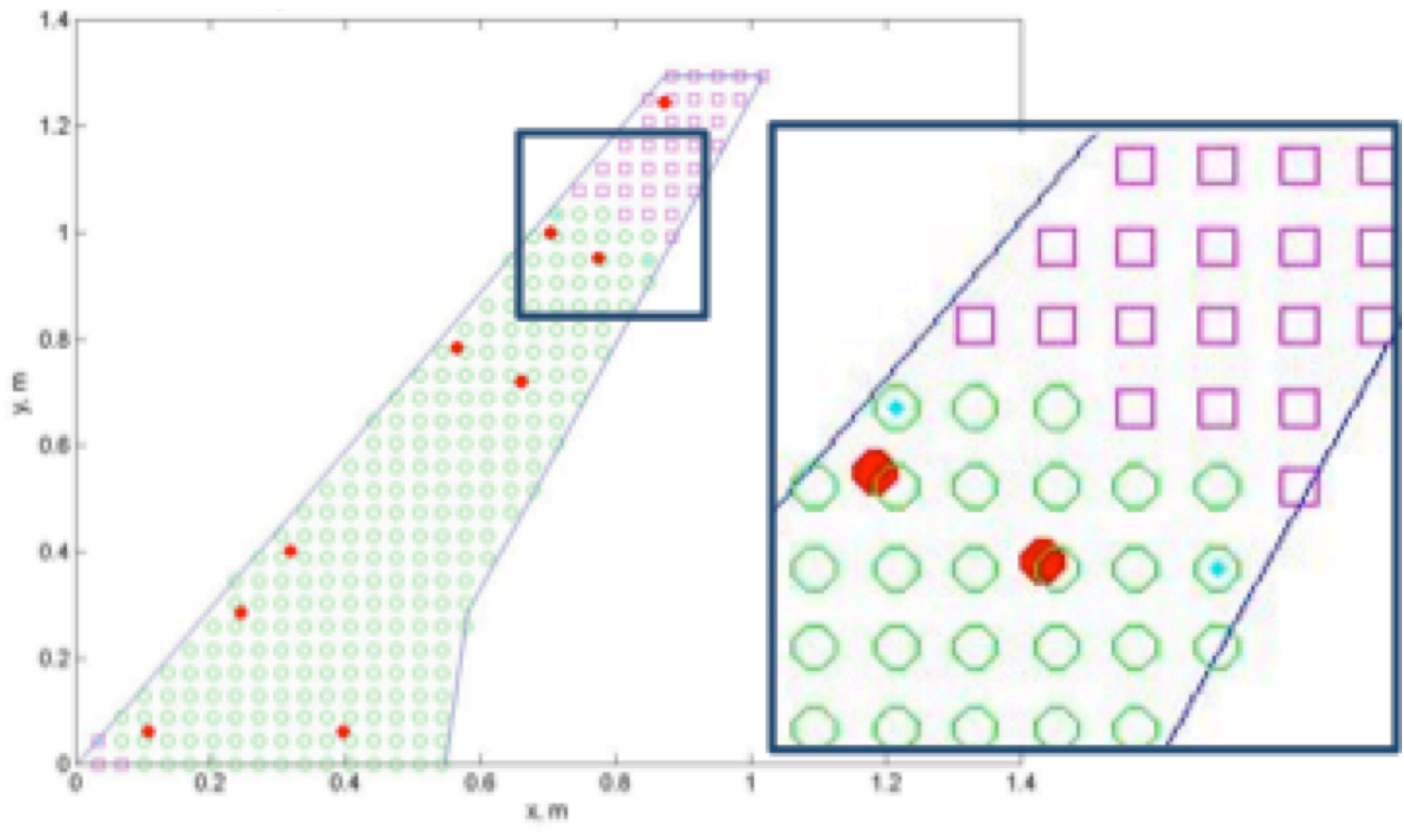

Figure 24. Node line of final modified FEM.

A comparison of the node lines for just the finite element modes for the second bending mode is shown in Figure 25. These results were generated from the mode shapes interpolated to the aerodynamic surface grid. The contour shading is shown for the second bending mode of the Final Modified FEM. The black line corresponds to the node line of the Original FEM and the red line corresponds to the location of the node line for the final modified FEM. The black symbol indicates the location of the reference accelerometer $\mathrm{Nr} 15 / 1$. The modifications to the FEM moved the second bending mode node line slightly inboard. The dashed lines show the locations of the chords of pressure transducers. 


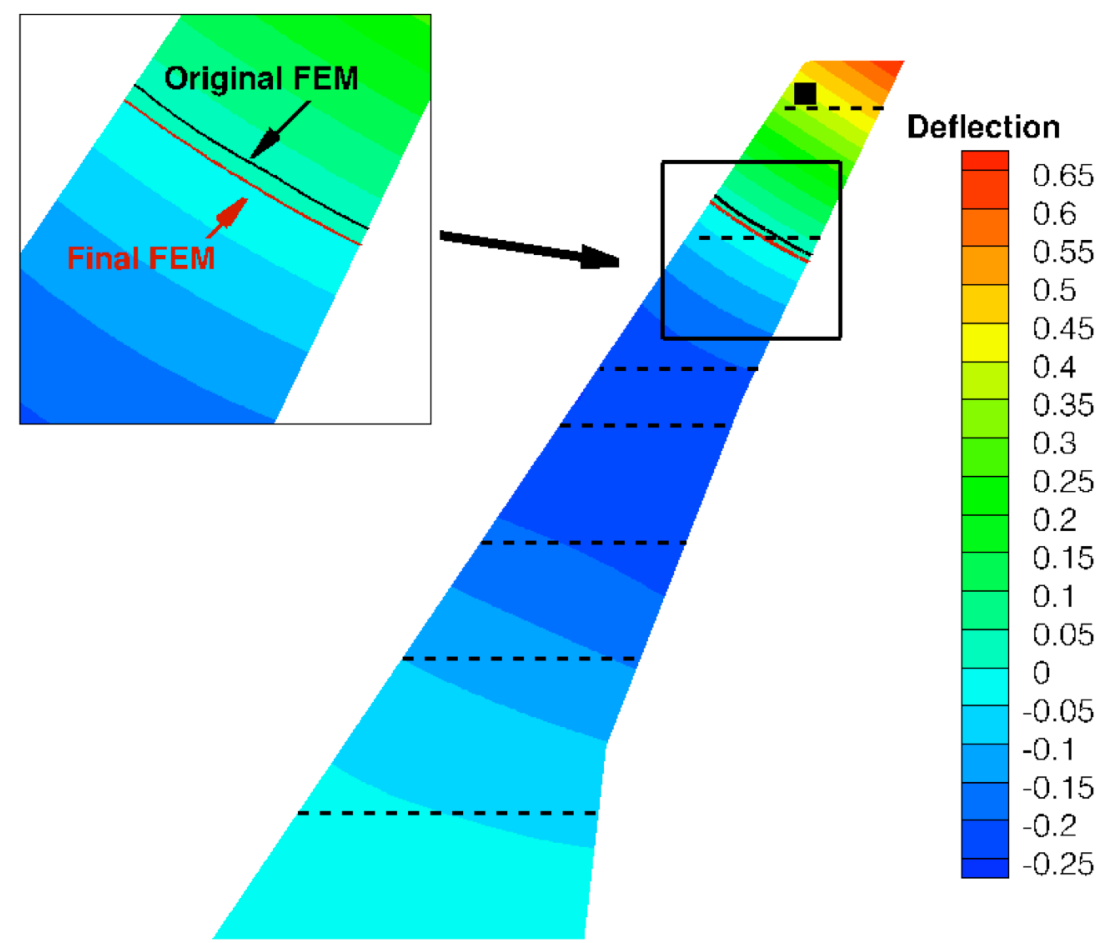

Figure 25. Comparison of nodelines of second bending mode interpolated to CFD grid.

\section{Computational Aeroelastic Process}

Computational aeroelastic results were generated for the HIRENASD configuration using the NASA Langleydeveloped computational fluid dynamics (CFD) software FUN3D ${ }^{20}$ at one of the static aeroelastic conditions of $\mathrm{M}=0.8, \mathrm{Re}_{\mathrm{c}}=7$ million, $\alpha=1.5$ degrees and one forced condition of the second bending mode at the same Mach number and Reynolds number comparing two different sets of finite element modes.

The details of the grids and parameters are provided in reference 22. The grid was identical between the two sets of CFD analyses and the grid chosen was the medium grid, with details published in the reference.

\section{A. Rigid Steady-Flow Analyses}

Solutions to the Reynolds-Averaged Navier-Stokes (RANS) equations were computed using the FUN3D flow solver. Turbulence closure was obtained using the Spalart-Allmaras one-equation model. The HIRENASD analyses presented in this paper were completed without a flux limiter. For the asymptotically steady cases under consideration, time integration was accomplished by an Euler implicit backwards difference scheme, with local time stepping to accelerate convergence. Most of the cases in this study were run for 10,000 iterations to achieve convergence of forces and moments to within $\pm 0.5 \%$ of the average of their last 1,000 iterations.

\section{B. Dynamic Analyses}

Dynamic analyses of the HIRENASD configuration required moving body and therefore grid motion capability. The grid deformation in FUN3D is treated as a linear elasticity problem. In this approach, the grid points near the body can move significantly, while the points farther away may not move at all. In addition to the moving body capability, the analysis of the HIRENASD configuration required dynamic aeroelastic capability. This capability is available in the FUN3D solver. ${ }^{20}$ For structural dynamics analysis, FUN3D is capable of being loosely coupled with an external finite element solver or in the case of the linear structural dynamics used in this study, an internal modal 
structural solver can be utilized. Modes were interpolated to the surface mesh using the method developed by Samareh. $^{21}$

The HIRENASD dynamic analysis was performed in a three-step process. First, the steady CFD solution was obtained for the rigid body. Next, a static aeroelastic solution was obtained by continuing the CFD analysis in a time accurate mode with a structural modal solver, allowing the structure to deform. A high value of structural damping (0.99) was used so the structure could find its equilibrium position with respect to the mean flow before the dynamic response was started. Finally, for the dynamic response, a user-specified modal motion was used. In this study, for harmonic perturbation, the modal displacement for mode $\mathrm{n}$ was computed as

$$
q_{n}=A_{n} \sin \left(\omega_{n} t\right)
$$

where $A_{n}$ is amplitude, $\omega_{n}$ is frequency, $t$ is time and $\mathrm{n}$ is the index of the second bending mode.

\section{Post Processing}

The dynamic comparison data selected for AePW were the magnitudes and phases of the frequency response functions (FRF). The FRF of principal interest were the pressure coefficients due to displacement at the $\mathrm{Nr} 15 / 1$ location normalized by the reference chord. This is the location of the accelerometer nearest the wing tip.

The details of this Fourier domain analysis process are provided in reference 22. The brief bottom line is that they were calculated using Power Spectral Densities and Cross spectral densities (PSD's and CSD's) which were computed using Welch's periodogram method. The block size was chosen to minimize the standard deviation among the periodograms of the peaks of the PSD and the overlap average was chosen to maximize the number of data blocks to reduce processing-based uncertainty.

The magnitude of the FRF is presented as:

$$
\left|\frac{C_{p}\left(f^{*}\right)}{Z / c_{\text {ref }}}\right| \text { vs } \frac{x}{c}
$$

In this paper the unsteady pressures were also integrated to obtain section lift and pitching moment. The real parts of the pressures were integrated at each span station corresponding to where the row of pressure sensors were located on the wind-tunnel mode shown in Figure 25.

\section{Computational Aeroelastic Results}

\section{A. Static - Aeroelastic Solution}

The static aeroelastic results were computed at $\mathrm{M}=0.8, \mathrm{Re}_{\mathrm{c}}=7$ million, an angle of attack of 1.5 degrees, The results showed indiscernable differences in the static pressure distributions using 30 flexible modes from each of the two FEMs. The static aeroelastic pressure distributions for the final FEM for both the upper and lower surfaces are shown in Figures 26 and 27, respectively. Since the CFD analyses were both conducted on identical grids the differences in the upper surface pressures between the two different CFD analyses as well as the differences in the static pressures of the lower surface could easily be computed. The differences are shown in Figures 28 and 29 for the upper and lower surface pressures respectively. 


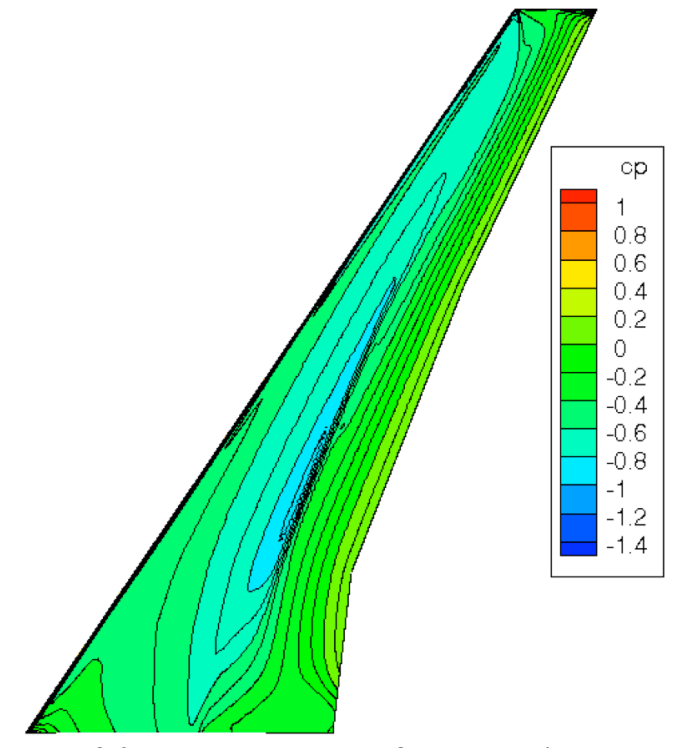

Figure 26. Upper surface static pressure distributions of the Final FEM.

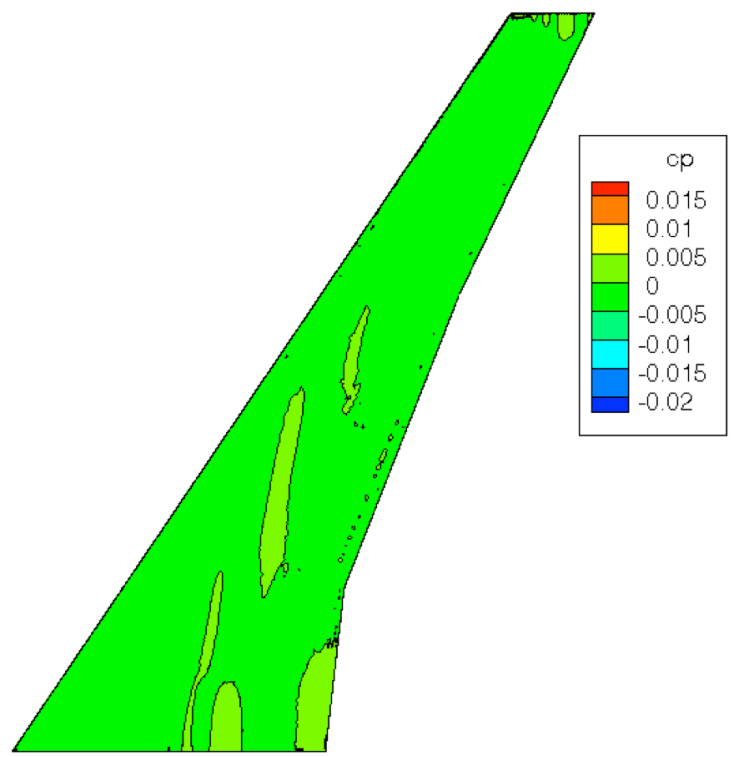

Figure 28 Difference in upper surface static pressures between Original and Final FEMs.

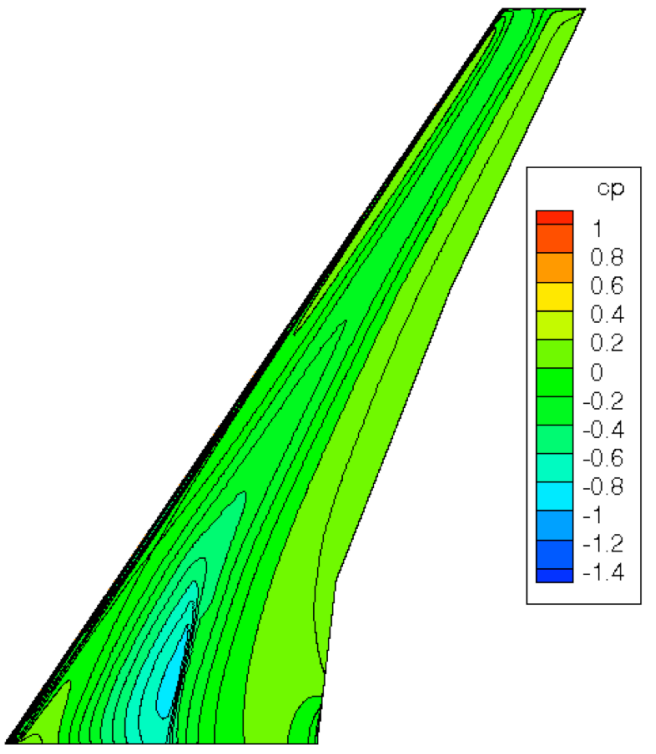

Figure 27. Lower surface static pressure distribution of the Final FEM.

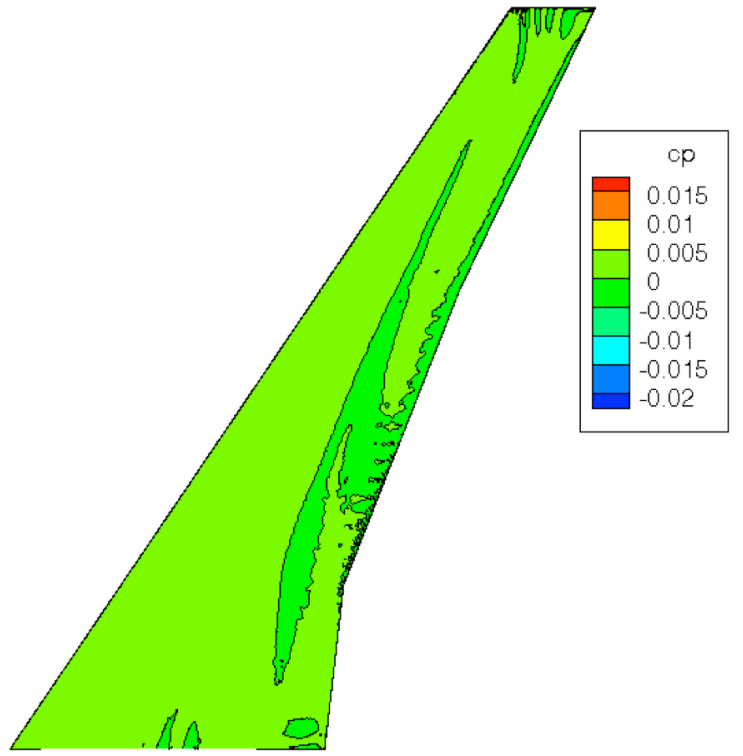

Figure 29. Difference in lower surface pressures between Original and Final FEMs.

\section{B. Comparisons of Unsteady CFD Results}

The HIRENASD experimental harmonic excitation tests were conducted to measure the interaction between the aerodynamics and the modal excitations of the wing. The forcing frequency for this case was $78.9 \mathrm{~Hz}$. Numerically the modal excitation was accomplished using equation 3.

The unsteady pressure frequency responses were calculated using the post processing methods described earlier. The magnitude, phase, real and imaginary parts were compared directly at each experimental span station and the differences were small but discernible. 
It was discussed in reference 5 and 6 that the dynamics of this problem are dominated by the shock oscillations on the upper surface. At the inboard-most span station on the lower surface, there is also an oscillating shock. The largest magnitude difference observed in the shock oscillation regions is on the order of $10 \%$; this occurring for the lower surface shock at the inboard span station. This is illustrated in Figure 30, by direct comparison of the two magnitude plots. The figure also shows the absolute value of the difference between these two data sets.

The differences on the upper surface in the region of the dynamic shock motion are considerably less. Analysis of pressure coefficient data for both upper and lower surface at seven span stations on the wing show larger percent differences for the leading and trailing edge dynamic responses. These larger differences, however, occur in areas where the overall dynamic response is low, inflating the percent differences.

There are other locations on the wing where the percent differences are larger but this is also due to the normalization by small numbers so the percent differences are inflated without physical signficance.

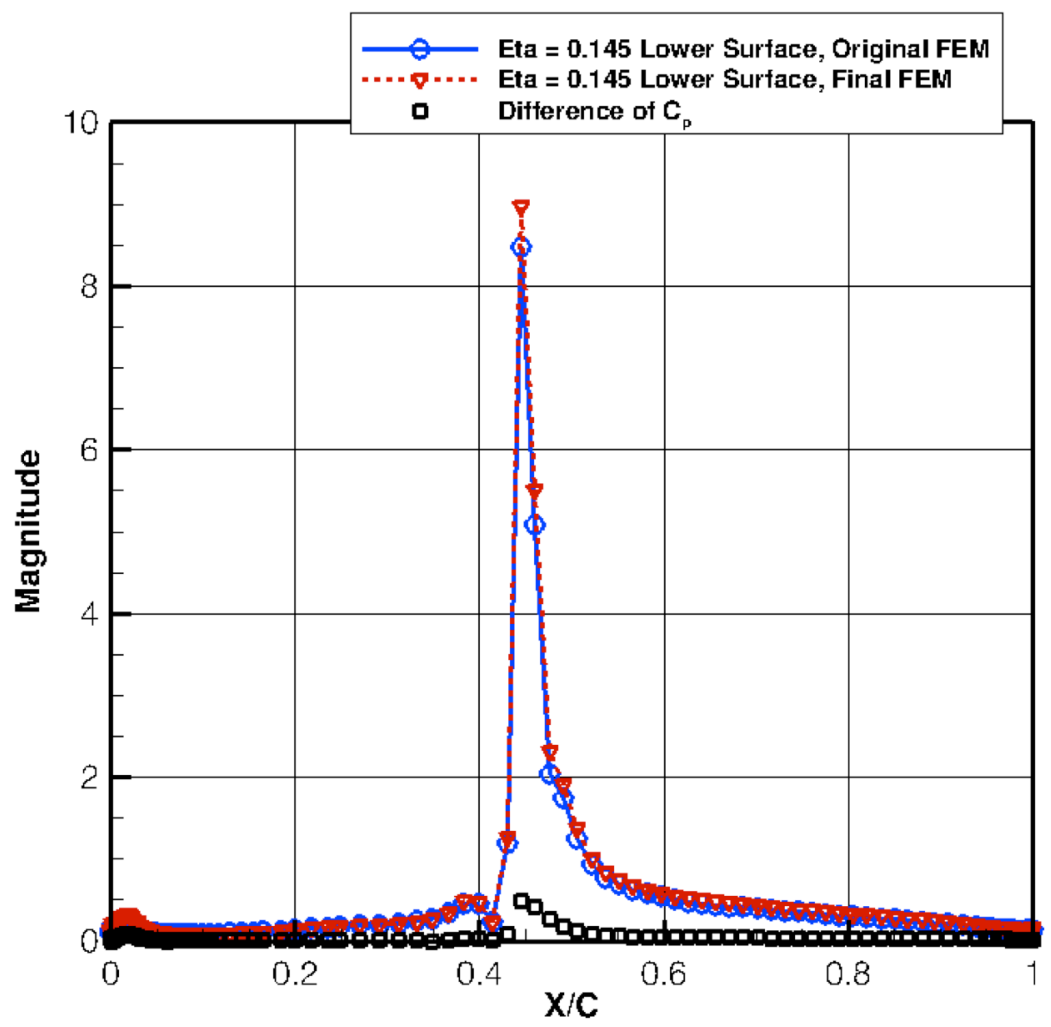

Figure 30. Magnitude of unsteady pressures and the difference of pressure coefficients, inboard span station, lower surface.

\section{Comparison of Unsteady Section Properties}

Comparisons of the section properties were used as another method to assess the impact of the modifications to the FEM.

The unsteady section lift and pitching moment were calculated by trapezoidal integration of the pressure distributions calculated from the CFD analyses. The lift was calculated from the difference in the integrated pressures of the lower surface minus the integrated pressures of the upper surface. The magnitude and phase of the section lift are shown in Figures 31 and 32, respectively. The final modified FEM yielded higher section lift than the Original FEM at the inboard stations and lower magnitude at the outboard stations. The section pitching moment coefficient about the balance location was calculated in a similar manner, (lower surface contribution upper surface contribution). These results are shown in Figures 33 and 34, respectively. The difference in the magnitude of the section pitching moment is almost neglible at all the span stations except for the most outboard span station. The difference in the phase of the section lift and pitching moment are greatest at the inboard station. 


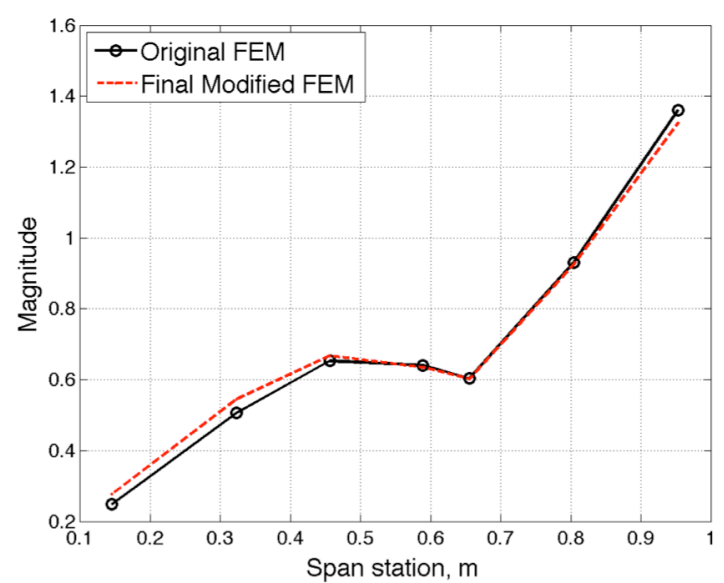

Figure 31 Comparison of magnitude of section lift.

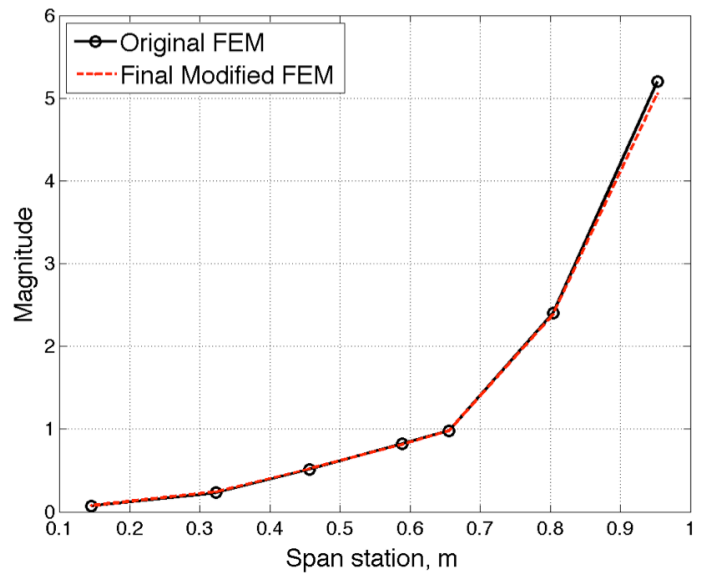

Figure 33. Comparison of magnitude of section pitching moment.

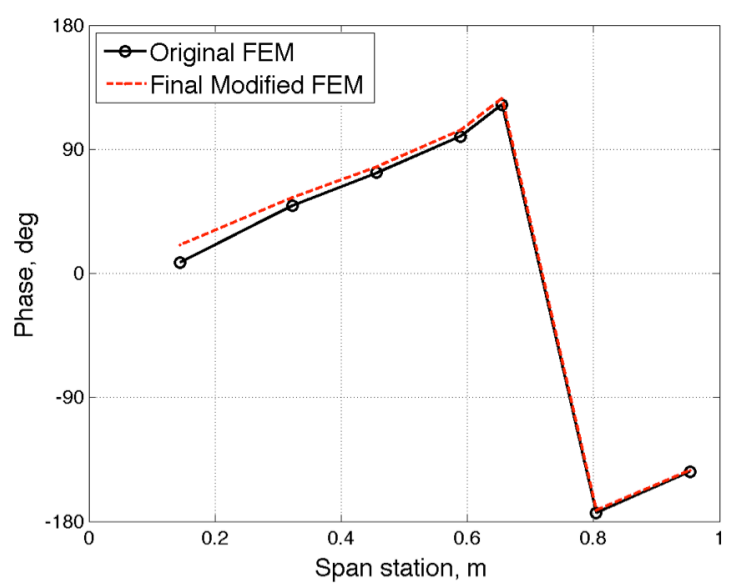

Figure 32 Comparison of Phase of section lift.

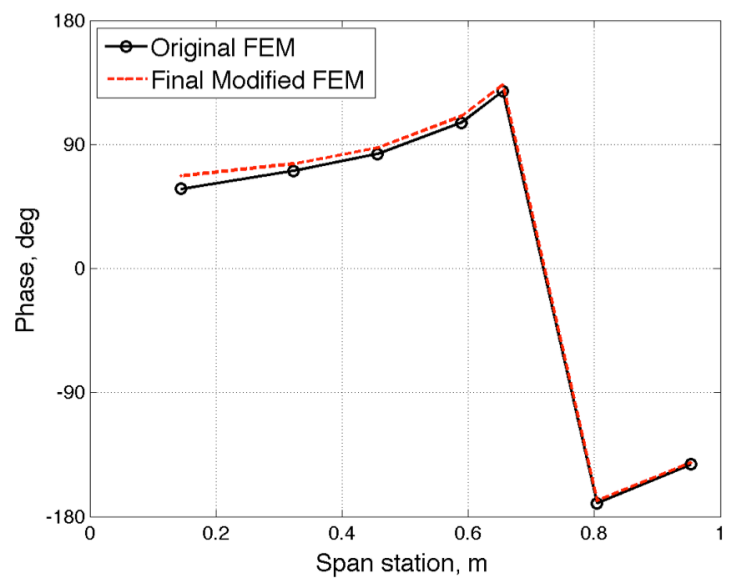

Figure 34. Comparison of phase of section pitching moment.

In order to assist in determining reasons for the differences in the unsteady pressure distributions, the mode shapes were examined more thoroughly by looking at the deflections of leading edge, trailing edge and twist from the entire FEM instead of just the accelerometer locations interpolated to the wing planform. The impact of the interpolation/extrapolation from nine geometric locations are compared with the direct measurements in the finite element model in Appendix A.

The small differences in the unsteady pressure distributions are the results of the small changes in the mode shapes between the two Finite Element Models.

\section{Conclusions}

The paper summarized the historical development of the enhanced HIRENASD structural model and compared CFD results for models using the original FEM as compared to the final enhanced FEM using the same aerodynamic theory and CFD code. The modifications to the Finite Element Models were validated by comparing modal frequencies, modal assurance criteria, comparing leading edge, trailing edge and twist of the wing compared with experiment and by performing steady and unsteady CFD analyses for one of the test conditions on the same grid, and identical processing of results. The modal assurance criteria showed improvement in the correlation of the first torsion and fourth bending mode without corruption of the other modes. There was a significant change in the frequency of the second bending mode with minor impact on the other modes. The largest difference in the 
frequency was due to the addition of the exciter system and balance. The second bending mode node line was shifted inboard and the fourth bending mode was now captured better in comparison with the experiment. The static aeroelastic results and the second bending forced excitation results did not change significantly by changing the FEM. The effort presented in this paper increased confidence in the structural dynamics representation. This was crucial to the Aeroelastic Prediction Workshop in that differences of the CFD results from different analysts as compared to experiment could then be clearly discussed in terms of aerodynamic differences and not structural modeling.

\section{Acknowledgments}

We wish to acknowledge Prof. Josef Ballmann and RWTH Aachen University for providing the solid-element models and additional information required to enhance these models to provide the best and most accurate model for the Aeroelastic Prediction Workshop.

\section{Appendix A. Limitation of modal interpolation using nine geometric locations}

This appendix shows the limitations of interpolation and extrapolation of the mode shape from nine locations to the entire wing. Figure 35 shows comparisons of the leading edge deflections, trailing edge deflections and twist for just the Final Modified FEM. The comparison of these deflections for the same finite element model show the difficulty in resolving the second bending mode deflections at the leading edge and trailing edge where extrapolation was required. The locations of the accelerometers with reference to the wing planform were shown in figures 11 and 22-24. The proximity of the measurement locations to the leading edge provided rather good extrapolation of the deflections to the leading edge except inboard of all the measurement locations.

The differences in the trailing edge deflections are significant inboard and outboard of the measurement locations where extrapolation was required. The differences are also significant at other span stations because of the lack of the $10^{\text {th }}$ accelerometer located inboard near the trailing edge. However to compare experimental modeshapes with modeshapes obtained analytically with the finite element model the only data that were available were the deflections at these nine accelerometer locations. The error due to the extrapolation and interpolation scheme therefore was present in all of the interpolations to the wing. The deflections and twist using the entire FEM for both the original FEM and the Final FEM are compared in Figure 36. There is very little difference between them.

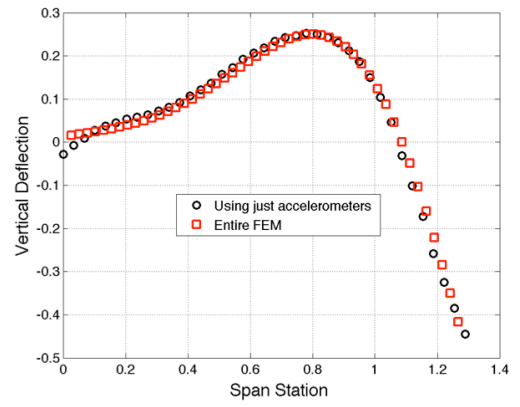

a. Vertical Deflection at LE.

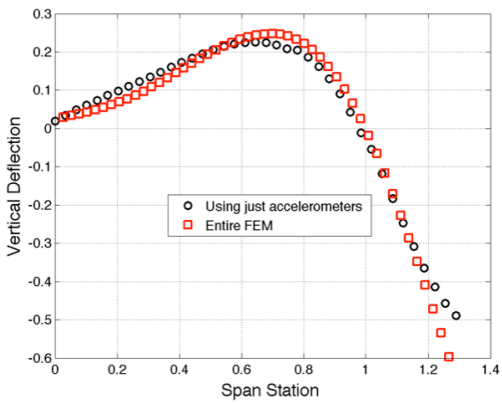

b. Vertical deflection at TE.

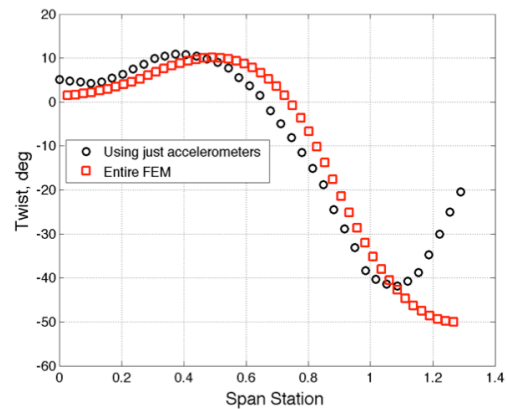

c. Wing twist.

Figure 35. Comparison of modeshape interpolation using 9 locations with output from entire FEM. 


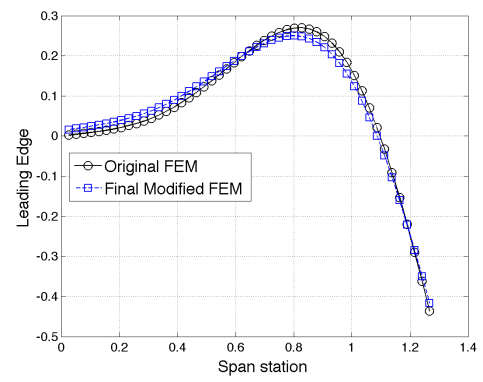

a. Vertical Deflection at LE.

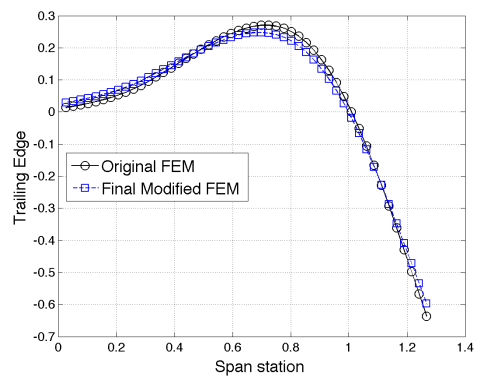

b. Vertical deflection at TE.

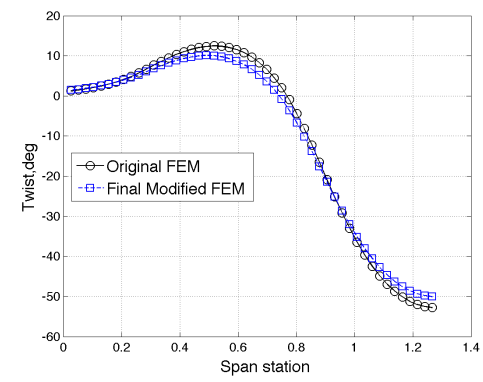

c. Wing Twist.

Figure 36. Comparison of Original FEM and Final Modified FEM using entire FEM.

\section{References}

1. Levy, David W., et al., "Summary of Data from the First AIAA CFD Drag Prediction Workshop," AIAA Paper AIAA2002-0841, Jan. 2002.

2. Rumsey, et al., "Summary of the First AIAA CFD High Lift Prediction Workshop," AIAA Paper AIAA-2011-0939, Jan. 2011.

3. Heeg, J, Chwalowski,P., Florance,J.P., Wieseman,C., Schuster, D. and Perry III, B. "Overview of the Aeroelastic Prediction Workshop", AIAA 2013-0783, presented at the 51st AIAA Aerospace Sciences Meeting; 7-10 Jan. 2013; Grapevine, TX.

4. Schuster, et al., Summary of Data and Findings from the First Aeroelastic Prediction Workshop, ICCFD7-3101, Seventh International conference on Computational Fluid Dynamics, Hawaii, July 9-13, 2012.

5. Schuster, D.M., Heeg, J., Wieseman, C.D., and Chwalowski, P., "Analysis of test case computations and experiments for the Aeroelastic Prediction Workshop," AIAA-2013-0788, presented at the 51st AIAA Aerospace Sciences Meeting; 7-10 Jan. 2013; Grapevine, TX.

6. Heeg, J., Chwalowski,P., Schuster, D., Dalenbring, M., "Overview and Lessons Learned from the Aeroelastic Prediction Workshop", AIAA paper, 54 ${ }^{\text {th }}$ AIAA SDM Conference, Boston, MA, April 2013 (submitted for publication)

7. Reimer, L., Braun, C., Chen, B.-H., Ballmann, J. "Computational Aeroelastic Design and Analysis of the HiReNASD Wind Tunnel Wing Model and Tests“, International Forum on Aeroelasticity and Structural Dynamics, IF-077, Stockholm. 2007.

8. Dafnis, A., Korsch, H., Buxel, C., Reimerdes, H.-G. "Dynamic Response of the HiReNASD Elastic Wing Model under Wind-Off and Wind-On Conditions", International Forum on Aeroelasticity and Structural Dynamics, IF-073, Stockholm. 2007.

9. Ballmann, J., et al., "Experimental Analysis of High Reynolds Number Aero-Structural Dynamics in ETW," AIAA Paper 2008-0841, Jan 2008.

10. Reimer, L., Boucke, A, Ballmann, J and Behr, M, Computational Analysis of High Reynolds Number Aero-structural Dynamics (HIRENASD) Experiments, IFASD-2009-130.

11. Korsch, H. ; Dafnis, A.; Reimerdes, Dynamic qualification of the HIRENASD elastic wing model, H.-G. Source: Aerospace Science and Technology, v 13, n 2-3, p 130-138, March 2009.

12. https://c3.nasa.gov/dashlink/projects/47/, January 2013.

13. RWTH Aachen website dedicated to HIRENASD, http://heinrich.lufmech.rwth-aachen.de/en/home.

14. MSC Software, Santa Ana, CA, http://www.msc.software.com/products.

15. MATLAB. A product of Mathworks. https://www.mathworks.com.

16. ARTeMIS,- Ambient Response Testing and Modal Identification Software (http://www.svibs.com).

17. Wieseman, C.D., Hoadley, S.T. and McGraw, S.M., On-line analysis capabilities developed to support the AFW Windtunnel tests, Journal of Aircraft, Vol. 32, No. 1, Jan-Feb 1995.

18. Phillips, A.W., Allemang, R.J, and Fladung. W.A., "The Complex Mode Indicator Function (CMIF) as a parameter estimation method." Proceedings-SPIE The International Society for Optical Engineering. Vol. 1. SPIE International Society for Optical,, 1998.

19. Allemang,R.J and Brown, D.L. "Experimental Modal Analysis and Dynamic Component Synthesis, Volume III. Modal Parameter Estimation" USAF: AFWAL-TR-87=3069, Volume III. pp 66-86.

20. Biedron, R. T. and Thomas, J.L., "Recent Enhancements to the FUN3D Flow Solver for Moving-Mesh Applications," AIAA Paper 2009-1360, Jan. 2009.

21. Samareh, J.A. "Discrete Data Transfer Technique for Fluid-Structure Interaction,” AIAA Paper 2007-4309, June 2997.

22. Chwalowski, P., Heeg, J., Wieseman, C., Florance, C. "FUN3D Analyses in Support of the First Aeroelastic Prediction Workshop", AIAA Paper 2013-0785, presented at the 51st AIAA Aerospace Sciences Meeting; 7-10 Jan. 2013; Grapevine, TX. 